(C) Institute of Mathematical Statistics, 2019

\title{
Gaussian Integrals and Rice Series in Crossing Distributions-to Compute the Distribution of Maxima and Other Features of Gaussian Processes
}

\section{Georg Lindgren}

\begin{abstract}
We describe and compare how methods based on the classical Rice's formula for the expected number, and higher moments, of level crossings by a Gaussian process stand up to contemporary numerical methods to accurately deal with crossing related characteristics of the sample paths.

We illustrate the relative merits in accuracy and computing time of the Rice moment methods and the exact numerical method, developed since the late 1990 s, on three groups of distribution problems, the maximum over a finite interval and the waiting time to first crossing, the length of excursions over a level, and the joint period/amplitude of oscillations.

We also treat the notoriously difficult problem of dependence between successive zero crossing distances. The exact solution has been known since at least 2000, but it has remained largely unnoticed outside the ocean science community.

Extensive simulation studies illustrate the accuracy of the numerical methods. As a historical introduction an attempt is made to illustrate the relation between Rice's original formulation and arguments and the exact numerical methods.
\end{abstract}

Key words and phrases: Computational statistics, distribution of maximum, Durbin's formula, excursion length distribution, first passage, independent interval assumption, level crossings, multivariate normal probabilities, period/amplitude distribution, Rice's formula, RIND program, statistical computation, stochastic process, successive crossing distance distribution, truncated normal moments, Wafo toolbox.

\section{CROSSING DISTRIBUTIONS-WHY AND HOW}

"In 1944 and 1945, S. O. Rice published a monumental study of noise, generally regarded to be the single most useful source of information about Gaussian noise" (Millman, 1984, page 41). The two papers (Rice 1944, 1945) were reprinted in Wax (1954), pages 133 294.

The strong words in the quote illustrate how it came about that abstract theoretical concepts, like Gaussian

Georg Lindgren is Professor emeritus, Mathematical Statistics, Lund University, Box 118, SE-221 00, Lund, Sweden (e-mail:georg@maths.lth.se). process, correlation function and power spectrum, linear and nonlinear filters, became common goods in one, very specialized, field of engineering, namely communication theory. Steve Rice was a member of the Bell Labs Mathematical and Statistical research center. Rice, quoting Wiener (1930), Khintchine (1934), and Cramér (1940) on Fourier representation of the covariance function, remarks (Rice, 1944, page 32) that "Khintchine and Cramér appear to be interested primarily in questions of existence, representation, etc., and do not stress the concept of the power spectrum". Middleton (1988) gives a personal history.

Rice's formula for the average number of level crossings in correlated Gaussian noise in continuous time 
is perhaps the most well known of the many original contributions in the two articles. The formula was presented as part of an investigation of the fluctuations in the length of the intervals between crossings, but it has earned its reputation as a fundamental property of stationary stochastic processes.

In wider perspectives, Rice's study of random noise in communication systems had far-reaching consequences outside communication engineering to lift the statistical methods beyond simple distributions, correlation, and energy spectra. Gaussian processes and Gaussian fields in linear and nonlinear filters were quickly accepted as standard tools in ocean engineering (StDenis and Pierson, 1953), aviation engineering (Miles, 1954), acoustics (Lyon, 1956), automatic control (Laning and Battin, 1956), mechanical engineering (Crandall, 1958), and many other fields of science and engineering. Blake and Lindsey (1973) give an overview of the early history, with a short follow up by Abrahams (1986). Kratz (2006) gives a more up to date and theoretical synthesis.

A recurring theme in the mentioned applications is the topographical features of a random function, such as the crossing intervals and excursion lengths, wave height and wave length, amplitude of fatiguecausing fluctuations in a mechanical load process. We have found more than two dozen scientific publications in the English language during 1950-1959 dealing with such features and half of them deal with the expected number of axis crossings and the distribution of axis crossing intervals. Slepian (1962) summarizes known results and gives many new ones, with strict proofs, including the "Slepian's comparison lemma" (Leadbetter, Lindgren and Rootzén, 1983, Theorem 7.4.2). Even today, Rice's approximations based on crossing moments continue to find new applications, i.a. in material science (Estrade, Iribarren and Kratz, 2012), laser physics and optics (Youssef, Munakata and Takeda, 1996), to mention a few. A survey of solved and unsolved crossing problems from science and technology was presented by Munakata (1998).

Rice's generalized crossing formulae are also used to approximate the distribution of the maximum value of a random process in time or in combined time and space. Crossing moments in two and three dimensions are simple to calculate (Cramér and Leadbetter, 1967, Section 10.6) and (Azaiis and Wschebor, 2009, Chapter 5), and they can give good results for extreme value distributions over short intervals.

We shall, in the present paper, describe how Rice's "in and exclusion" series (Rice, 1945, equation (3.411)), are replaced by Durbin formula (Durbin, 1985), later refined by Rychlik (1987a, 1990), to give the exact "first crossing" distribution. Highly efficient algorithms for high-dimensional normal integration, developed by i.a. Genz (1992, 2012), Azaïs and Genz (2013), and Brodtkorb (2006), have made the theoretical Durbin's formula useful, and we shall illustrate how these are implemented in dedicated and simpleto-use software for computation of topographical distributions in Gaussian processes.

In Section 2, we summarize and comment on Rice's original arguments around three topographical themes, the expected number of axis crossings, the distribution of the interval between, and number of crossings, and the distribution of local extremes. We emphasize their relations to modern terminology and formulations.

The next three sections intend to show how the Rice series and the modern computational tools can be used on three types of general statistical problems. The focus is more on the flexibility and generality of the different methods and less on the formal complexity and difficulties of the numerical computations, which are well described in the cited works (Genz, 1992, 2012; Azaiis and Genz, 2013; Brodtkorb, 2006). We deal with single-parameter problems only, leaving the very different area of extremes of Gaussian fields outside the treatment.

Section 3 deals with the distribution of two related quantities that can be observed in a continuous stochastic process: the time from start to the first upcrossing of a fixed level, and the maximum value over a finite interval. We discuss the software by Brodtkorb and Genz, for calculating the distribution of the maximum, and compare the results with bounds based on Rice's crossings formula. The applications are plenty, just to mention a few from statistics, multiple tests and confidence bands in regression (Rychlik, 1992a; Efron, 1997; Azaïs and Genz, 2013; Azaïs, De Castro and Mourareau, 2018), statistical machine learning and Gaussian process regression (Rasmussen and Williams, 2006), as well as applications in science and engineering.

Section 4 deals with the statistical distribution of the length of excursions over a critical level, and with the dependence between successive zero crossings intervals, one of Rice's original problems. We compare the Rice series solutions with the exact solutions based on Gaussian integration, with regards to generality and computational efficiency. A new numerical algorithm is introduced for exact computation of the joint distribution of successive zero crossing intervals. 
In Section 5, we give examples of a more complicated characteristic of a stationary Gaussian process, the distribution of wave amplitude and period. This is a quantity of great importance in mechanical and ocean engineering, regularly employed in fatigue analysis and marine safety studies.

An appendix contains details about the example processes, technical details about the computations, and a presentation of the computational tool, the RIND function in the Wafo toolbox (Brodtkorb et al., 2000; WAFO-group, 2017a). Matlab code and instructions can be found in the Supplementary material, Lindgren (2019).

\section{RICE'S FORMULA FOR AXIS CROSSINGS AND EXCURSIONS}

In the second of the two papers, Rice (1945) introduces i.a. three themes of particular interest for the present study: the expected number of axis crossings, the distribution of the interval between and the number of crossings, and the distribution of local extremes.

\section{1 "Expected Number of Zeros" (Rice, 1945, Section 3.3)}

Rice's formula for the expected number of level crossings per time unit in a stationary stochastic process does not occupy any prominent place in Rice's pioneering work. Rather, it is expressed as the probability that a differentiable stochastic process $X(t)$, stationary or not, will have a zero crossing in a time interval $\tau, \tau+\mathrm{d} \tau$ (Rice, 1945, equation (3.3-2)).

RICE'S FORMULA FOR CROSSINGS. The probability that the (differentiable) process $X(t)$ has a zero crossing in $\tau, \tau+\mathrm{d} \tau$ is equal to

$$
\nu_{0}(\tau) \mathrm{d} \tau=\mathrm{d} \tau \int_{-\infty}^{\infty}|y| p(0, y ; \tau) \mathrm{d} y,
$$

where $p(u, y ; \tau)$ is the joint probability density of $X(\tau)$ and $X^{\prime}(\tau)$.

Rice's arguments do not require that $X(t)$ is stationary. On the contrary, he argues from a "random curve", generated by random variables $a_{1}, a_{2}, \ldots, a_{N}$,

$$
y=F\left(x ; a_{1}, a_{2}, \ldots, a_{N}\right) .
$$

In the vein of Kac (1943), Rice presents the formula for the expected number of solutions to the equation $F(x)=0$ as the integral of the crossing intensity $v_{0}(x)$, which obviously may depend on $x$. Rainal (1988) gives an interesting and personal history of the origin of Rice's formula.

Rice applies the crossings formula to the sum of random harmonics and arrives at the stationary Gaussian form for the expected number of upcrossings of a level $u$ per time unit (Rice (1945), equation (3.3-15)),

"Rice's formula"

$$
v_{u}^{+}=e^{-u^{2} / 2 \psi_{0}} \frac{1}{2 \pi}\left[-\frac{\psi_{0}^{\prime \prime}}{\psi_{0}}\right]^{1 / 2},
$$

assuming the mean is zero and with $\psi_{t}$ denoting the covariance function.

\section{2 "The Distribution of Zeros" (Rice, 1945, Section 3.4)}

For the distribution of crossing intervals and the number of zeros Rice acknowledges the difficulty and suggests a partial solution and a hint for an approximating scheme.

The Rice inclusion-exclusion series (Rice, 1945, equation (3.4-11)) for the probability density of the length of axis crossing intervals is a Bonferroni type series. It reads as follows, in Rice's arguments and notation and with slightly modified formulation, valid for a stationary process.

RICE'S IN- AND EXCLUSION SERIES FOR EXCURSIONS. Consider the class of sample curves having a zero at $t=0$ and compute the functions $p_{0}(\tau)$, $p_{1}(r, \tau), p_{2}(r, s, \tau)$, etc., associated with this class, where:

- $p_{0}(\tau) \mathrm{d} \tau\left(=v_{0}(\tau) \mathrm{d} \tau\right)$ is the probability the curve having a zero in $\mathrm{d} \tau$; (i.e., in $\tau, \tau+\mathrm{d} \tau$ ),

- $p_{1}(r, \tau) \mathrm{d} \tau \mathrm{d} r$ is the probability the curve having ze$\operatorname{ros}$ in $\mathrm{d} \tau$ and $\mathrm{d} r$,

- $p_{2}(r, s, \tau) \mathrm{d} \tau \mathrm{d} r \mathrm{~d} s$ is the probability the curve having zeros in $\mathrm{d} \tau, \mathrm{d} r$, and $\mathrm{d} s$,

- etc.

The method then leads to an expression for $P_{0}(\tau) \mathrm{d} \tau$, the probability of having a zero at 0 and a zero in $\tau, \tau+\mathrm{d} \tau$ but none between 0 and $\tau$. It is, without the $\mathrm{d} \tau$ factor,

$$
\begin{aligned}
P_{0}(\tau)= & p_{0}(\tau)-\frac{1}{1 !} \int_{0}^{\tau} p_{1}(r, \tau) \mathrm{d} r \\
& +\frac{1}{2 !} \int_{0}^{\tau} \int_{0}^{\tau} p_{2}(r, s, \tau) \mathrm{d} r \mathrm{~d} s \\
& -\frac{1}{3 !} \int_{0}^{\tau} \int_{0}^{\tau} \int_{0}^{\tau} p_{3}(r, s, t, \tau) \mathrm{d} r \mathrm{~d} s \mathrm{~d} t \\
& +\cdots
\end{aligned}
$$

The restriction to "the class of sample curves having a zero at $t=0$ " does not imply that the probabilities in (1) are conditional probabilities, "given the process 
TABLE 1

$\begin{array}{ll}v_{u}^{+}(t), v_{u}^{+}(X, t) & \text { upcrossing intensity of level } u \text { at time } t \text { by process } X(\cdot) \\ v_{u}^{+-}(s, t) & \text { bivariate intensity of upcrossing at } s \text { and downcrossing at } t \\ v_{u}^{m+}(\boldsymbol{t}) & m \text {-variate upcrossing intensity at } t=\left(t_{1}, \ldots, t_{m}\right) \\ \alpha^{u}, \alpha^{u}(t) & \text { expected number of } u \text {-upcrossings in }[0, t] \\ \alpha_{m}^{u}, \alpha_{m}^{u}(t) & m \text { th factorial moment of number of } u \text {-upcrossings in interval }[0, t] \\ \widetilde{v}_{u}^{m+}(\boldsymbol{t}) & m \text {-variate upcrossing intensity at } \boldsymbol{t}=\left(t_{1}, \ldots, t_{m}\right) \\ & \text { when sample path satisfies an initial condition, } \\ \widetilde{\alpha}_{m}^{u}(t) & \text { e.g. } X(0)<u \text { or } X(0)=u, \text { upcrossing } \\ X(\boldsymbol{t})=\boldsymbol{u} \text { and } X_{\boldsymbol{t}}=\boldsymbol{u} & m \text { th factorial moment for selected sample paths } \\ X_{I} \leq u & X\left(t_{k}\right)=u \text { for } k=1, \ldots, m, \\ \mu_{u}^{+}(X, t) & X(t) \leq u \text { for } t \in I, \\ & u \text {-upcrossing intensity at } t \text { with } X_{[0, t]} \leq u .\end{array}$

takes the value 0 at $t=0$ ", and not any other value. Rather, it means that we have selected one of the time points where the process crosses the zero level, and called it $t=0$. The conditional probability density is obtained by dividing throughout by $v_{0}(0)$.

Rice backed away from the difficult integrals involved in the higher-order approximations. Interestingly enough, he mentions (Rice, 1945, page 70) the possibility to amend the condition leading to $p_{0}(\tau)$ with the condition that the process has constant sign at equally spaced points between 0 and $\tau$. He concludes that also these integrals are hard to evaluate; $\mathrm{cf}$. Durbin's formula, Fact 2 in Section 3.1.

EXAMPLE 1 (Up- to downcrossing interval). We illustrate the use of the in- and exclusion formula to find a partial solution to the density of the interval between an upcrossing and the following downcrossing of the mean value level. Details about the numerical computations are given in Section 4. Table 1 presents some selected notations used in the paper.

In Rice's words, we first seek the probability that the process passes through zero in the interval $\tau, \tau+d \tau$ with a negative slope, when it is known that it passes through zero at $\tau=0$ with a positive slope. Let, with Rice (1945), equation (3.4-12),

$$
\begin{aligned}
& v_{0}^{+-}(s, t) \mathrm{d} s \mathrm{~d} t \\
& =\mathrm{d} s \mathrm{~d} t \int_{y_{1}=0}^{\infty} \int_{y_{2}=-\infty}^{0} y_{1}\left(-y_{2}\right) \\
& \quad \cdot p\left(0,0, y_{1}, y_{2} ; s, t\right) \mathrm{d} y_{1} \mathrm{~d} y_{2}
\end{aligned}
$$

be the probability that the process has a zero upcrossing in $\mathrm{d} s$ and a zero downcrossing in $\mathrm{d} t$. We use here the signed superscript notation that became standard in applications of the "inclusion and exclusion" series for crossing analysis (Longuet-Higgins, 1962). For exam- ple, in (1),

$$
\begin{aligned}
& p_{1}(r, \tau) \\
& \quad=v^{ \pm \pm}(r, \tau) \\
& \quad=\int_{-\infty}^{\infty} \int_{-\infty}^{\infty}\left|y_{1}\right|\left|y_{2}\right| p\left(0,0, y_{1}, y_{2} ; r, \tau\right) \mathrm{d} y_{1} \mathrm{~d} y_{2} .
\end{aligned}
$$

Expressed in terms of a conditional expectation of

$$
\begin{aligned}
X^{\prime}(0)^{+} & =\max \left(0, X^{\prime}(0)\right), \\
X^{\prime}(t)^{-} & =-\min \left(0, X^{\prime}(0)\right) \geq 0,
\end{aligned}
$$

we can write the intensity in (2) as

$$
\begin{aligned}
v_{0}^{+-}(0, t)= & \mathrm{E}\left[X^{\prime}(0)^{+} X^{\prime}(t)^{-} \mid X(0)=X(t)=0\right] \\
& \cdot f_{X(0), X(t)}(0,0),
\end{aligned}
$$

which is the form we shall use in the examples. Rice computes $v_{0}^{+-}(0, \tau) / v^{+}(0)$ as an explicit first approximation to the up-to-down zero-crossing distance in a stationary Gaussian process.

To get more terms in the series, we have to add extra downcrossings between 0 and $t$,

$$
\begin{aligned}
& v_{0}^{+--}(0, s, t) \\
&= \mathrm{E} \\
& {\left[X^{\prime}(0)^{+} X^{\prime}(s)^{-} X^{\prime}(t)^{-} \mid X(0)\right.} \\
&=X(s)=X(t)=0] \\
& \cdot f_{X(0), X(s), X(t)}(0,0,0), \\
& v_{0}^{+---}\left(0, s_{1}, s_{2}, t\right) \\
&=\mathrm{E}\left[X^{\prime}(0)^{+} X^{\prime}\left(s_{1}\right)^{-} X^{\prime}\left(s_{2}\right)^{-} X^{\prime}(t)^{-} \mid X(0)\right. \\
&\left.=X\left(s_{1}\right)=X\left(s_{2}\right)=X(t)=0\right] \\
& \cdot f_{X(0), X\left(s_{1}\right), X\left(s_{2}\right), X(t)}(0,0,0,0) .
\end{aligned}
$$

The excursion time density approximated with the first three terms in the Rice in- and exclusion series is 


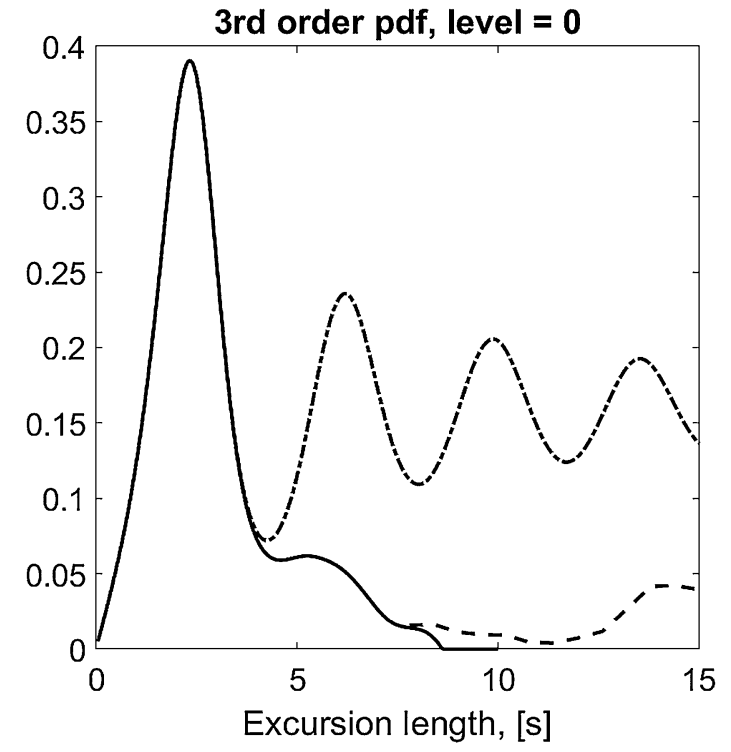

FIG. 1. Rice series approximations to the axis excursion time density for low-frequency white Gaussian noise. First order (dashdotted) and third order (dashed) overestimates the density, second order (solid) under-estimates it.

then

$$
\begin{aligned}
f_{T}(t) \approx & \frac{1}{v_{0}^{+}(0)}\left\{v_{0}^{+-}(0, t)-\int_{s=0}^{t} v_{0}^{+--}(0, s, t) \mathrm{d} s\right. \\
& \left.+\iint_{0<s_{1}<s_{2}<t} v_{0}^{+---}\left(0, s_{1}, s_{2}, t\right) \mathrm{d} s_{1} \mathrm{~d} s_{2}\right\} .
\end{aligned}
$$

Figure 1 shows the over- and underestimation of the excursion density with one, two, and three terms in the series. The process is low-frequency Gaussian white noise with variance one and mean excursion length $\pi \approx 3.14$. In Section 4.4, we will see the exact density, Figure 7.

\section{3 "Distribution of Local Maxima" (Rice, 1945, Section 3.6)}

Rice (1945), Section 3.6, presented a result of quite some practical interest, namely the distribution of the height of local maxima (and minima) of a normal process.

RICE'S DISTRIBUTION FOR LOCAL MAXIMA. Let $y=F\left(x ; a_{1}, \ldots, a_{N}\right)$ be a random curve. If suitable conditions are satisfied, the probability that $y$ has a maximum in the rectangle $\left(x_{1}, x_{1}+\mathrm{d} x_{1}, y_{1}, y_{1}+\mathrm{d} y_{1}\right)$, $\mathrm{d} x_{1}$ and $\mathrm{d} y_{1}$ being of the same order of magnitude, is

$$
-\mathrm{d} x_{1} \mathrm{~d} y_{1} \int_{-\infty}^{0} \zeta p\left(y_{1}, 0, \zeta\right) \mathrm{d} \zeta
$$

and the expected number of maxima of $y$ in $a \leq$ $x \leq b$ is obtained by integrating this expression over the range $-\infty<y_{1}<\infty, a \leq x_{1} \leq b$. Here $p(\xi, \eta, \zeta)$ is the probability density function of $\xi=F\left(x_{1} ; a_{1}, \ldots\right.$, $\left.a_{N}\right)$ and the first- and second-order derivatives of $F(x)$ at $x=x_{1}$.

Applying the formula to a Gaussian process, Rice derives the explicit density for the height of a maximum selected at random from the universe of maxima. Rice's formulation is actually very close to the frequency interpretation of the horizontal window conditioning by $\mathrm{Kac}$ and Slepian (1959). The beauty of the result is not in the analytical form of the density (see Lindgren, 2013, equation (8.37)) but in its probabilistic representation.

RICE'S REPRESENTATION OF LOCAL MAXIMA. The distribution of the height of a local maximum $X\left(t_{\max }\right)$ in a Gaussian process with variance $\sigma_{X}^{2}$ is equal to the distribution of a weighted sum of a standard Rayleigh variable $R$ and a standard normal variable $U$,

$$
X\left(t_{\max }\right) \stackrel{\mathcal{L}}{=} \sigma_{X}\left\{\sqrt{1-\varepsilon^{2}} R+\varepsilon U\right\},
$$

where $\epsilon=\sqrt{1-\sigma_{X^{\prime}}^{2} /\left(\sigma_{X} \sigma_{X^{\prime \prime}}\right)}$ is a measure of the width of the spectral density of the process.

\section{MAXIMUM AND THE FIRST LEVEL CROSSING}

We start our exposé of Rice's formula and its extensions with the simplest, and perhaps most important, of applications, namely how to approximate the distribution of the maximum of a differentiable Gaussian process over a bounded interval. We first give the modern formulation of the Kac-Rice formula for the intensity of level upcrossings, and the related formula by Durbin (1985), extended by Rychlik (1987a), for the exact intensity of first upcrossing time and for the maximum distribution. In subsequent subsections, we present and discuss the two available alternatives to compute the distribution: the Rice method with alternating Rice series of factorial moments up to third order, and the numerical method based on numerical integration of Gaussian distributions.

\subsection{Rice's and Durbin's Formulae}

Let $\{X(t), t \geq 0\}$ denote a stochastic process, not necessarily stationary, that satisfies some quite natural conditions (see Azaïs and Wschebor, 2009, Chapter 3). Specifically, we assume that the sample paths are continuously differentiable with nondegenerate joint density $f_{X(t), X^{\prime}(t)}(x, u)$, and that, for any fixed level $u$ and finite interval $I$, there are no points in $I$ where $X(t)=u, X^{\prime}(t)=0$. This, of course, implies that each crossing is either a strict upcrossing or a strict down- 
crossing. Write

$$
M_{t}=\max _{0 \leq s \leq t} X(s), \quad U_{u}(t)=U_{u}(t ; X),
$$

for the maximum over $[0, t]$ and the number of $u$ upcrossings in $[0, t]$, respectively.

FACT 1 (Rice's formula). The intensity of $u$ upcrossings by $X(t)$ at time $t$ is

$$
v_{u}^{+}(X, t)=\mathrm{E}\left[X^{\prime}(t)^{+} \mid X(t)=u\right] f_{X(t)}(u),
$$

and the expected number of upcrossings in $[0, t]$ is the integral

$$
\begin{aligned}
\alpha_{1}^{u}(X, t) & =\int_{0}^{t} v_{u}^{+}(X, s) \mathrm{d} s \\
& =\int_{0}^{t} \mathrm{E}\left[X^{\prime}(s)^{+} \mid X(s)=u\right] f_{X(s)}(u) \mathrm{d} s .
\end{aligned}
$$

For a stationary Gaussian process with mean 0 , $\mathrm{V}[X(t)]=\lambda_{0}, \mathrm{~V}\left[X^{\prime}(t)\right]=\lambda_{2}$,

$$
v_{u}^{+}=\frac{1}{2 \pi} \sqrt{\lambda_{2} / \lambda_{0}} \exp \left\{-u^{2} / 2 \lambda_{0}\right\} .
$$

As anticipated by Rice, the intensity of first crossing can be obtained by adding conditions on the process in the interval preceding the crossing, for example, that $X(s)$ does not have any $u$-upcrossings before $t$. To formulate an exact relationship, we need to incorporate the starting value, and introduce the indicator function that the process starts below $u$ and has no $u$ upcrossings in $(0, t)$,

$$
\mathbf{1}_{u}(X, t)=\mathbf{1}_{\{X(s) \leq u \text { for } 0 \leq s \leq t\}} .
$$

This leads ultimately to a modified Durbin's formula for the restricted first upcrossing intensity, given in the following form by Rychlik (1987a), Theorem 2.

FACT 2 (Durbin's formula). The intensity at time $t$ of first $u$-level upcrossing by $X(t)$ is

$$
\begin{aligned}
& \mu_{u}^{+}(X, t) \\
& \quad=\mathrm{E}\left[\mathbf{1}_{u}(X, t) X^{\prime}(t)^{+} \mid X(t)=u\right] f_{X(t)}(u) .
\end{aligned}
$$

FACT 3 (The record method (Rychlik, 1990)). The relation between the maximum tail distribution $\mathrm{P}\left(M_{t}>\right.$ $u)=1-\mathrm{E}\left[\mathbf{1}_{u}(X, t)\right]$ and the restricted first upcrossing intensity $\mu_{u}^{+}(X, t)$ is

$$
\begin{aligned}
\mathrm{P}\left(M_{t}\right. & >u) \\
= & \mathrm{P}(X(0)>u) \\
& \quad+\int_{0}^{t} \mathrm{E}\left[\mathbf{1}_{u}(X, s) X^{\prime}(s)^{+} \mid X(s)=u\right] f_{X(s)}(u) \mathrm{d} s,
\end{aligned}
$$

representing the fact that either the process starts above $u$, or it starts below $u$ and has a first $u$-upcrossing before $t$.
The normalized function

$$
\tilde{f}_{T_{u}}(t)=\frac{1}{\mathrm{P}(X(0) \leq u)} \mu_{u}^{+}(X, t), \quad t>0,
$$

is the density of time to first upcrossing for sample paths that start below $u$.

\subsection{Maximum of a Stationary Gaussian Process; Simple Rice Method}

Due to its simplicity, the Rice method has found wide applications in engineering and science. It simply bounds the probability of high values in a continuous process by means of the expected number of level crossings, which involves the joint distribution of the process and its derivative.

The upper Rice bound for the maximum tail reads

$$
\begin{aligned}
\mathrm{P}\left(M_{t}>u\right) \leq & \mathrm{P}(X(0) \geq u)+\mathrm{P}\left(U_{u}(t)>0\right) \\
\leq & \mathrm{P}(X(0) \geq u)+\mathrm{E}\left[U_{u}(t)\right] \\
= & \mathrm{P}(X(0) \geq u) \\
& \quad+\int_{s=0}^{t} \int_{z=0}^{\infty} z f_{X(s), X^{\prime}(s)}(u, z) \mathrm{d} z \mathrm{~d} s .
\end{aligned}
$$

If $X(t)$ is stationary, the bound is $\mathrm{P}\left(M_{t}>u\right) \leq$ $\mathrm{P}(X(0) \geq u)+t v_{u}^{+}(X, 1)$ and, explicitly for a stationary Gaussian process,

$$
\begin{aligned}
\mathrm{P}\left(M_{t}>u\right) \leq 1 & -\Phi\left(\frac{u-m_{X}}{\sigma_{X}}\right) \\
& +\frac{t}{2 \pi} \frac{\sigma_{X^{\prime}}}{\sigma_{X}} \exp \left\{-\frac{\left(u-m_{X}\right)^{2}}{\sigma_{X}^{2}}\right\} .
\end{aligned}
$$

We will see, in Section 3.4, that the Rice bound for the maximum cdf can be very accurate: as a rule of thumb, above the upper tenth percentile of the distribution.

\subsection{Calculation of the Maximum Distribution by Gaussian Integrals}

To be useful for numerical calculations, the "infinite dimensional" expectation $\mathrm{E}\left[\mathbf{1}_{u}(X, s) X^{\prime}(s)^{+} \mid X(s)=\right.$ $u$ ] in (3) has to be replaced by a finite dimensional one, before it is integrated over time. For a smooth Gaussian process over a short interval, one could hope that few points would suffice; Arellano-Valle and Genton (2008) give an example of how to proceed. A long interval or a nonstationary mean/covariance function should require a large and sometimes dense point grid, and lead to almost singular covariance matrices. Long computation times and numerical complications should be expected.

The first algorithm that had a potential to develop into a general tool for fast and accurate computation of the distribution of the maximum of a smooth Gaussian 
process over a finite interval seems to have been published by Genz $(1992,1993)$. The algorithm was based on an ordered Cholesky factorization of the covariance matrix (as all subsequent algorithms); it was later improved by Genz and Kwong (2000).

Another line of development was followed by Rychlik (1992b) and Podgórski, Rychlik and Machado (2000), who used an explicit regression technique to select the integration dimensions (Lindgren and Rychlik, 1991). This regression technique is very similar to and plays the same role in the integration algorithm as the Gaussian processes regression to reduce uncertainty in modern Bayesian Machine learning; see, for example, O'Hagan (2006), Rasmussen and Williams (2006).

Brodtkorb (2006) combined the technique in Genz (1992), Genz and Kwong (2000) with the regression technique. Together with removal of redundant variables and truncation of the Cholesky factorization, this led to additional improvements in speed and accuracy.

Implementations of the various dialects of the GenzBrodtkorb algorithms exist, either as "stand-alone" packets or as parts of general projects. The MATLAB function RIND is part of the Wafo toolbox; WAFOgroup (2017a, 2017b). It is built on compiled FORTRAN and $\mathrm{C}++$ routines and is very fast. There is also a PYTHON implementation for Linux systems. The MATLAB package MAGP written by Mercadier (2006a) is available at Mercadier (2006b), and is based on RIND.

Genz (2012) presents a list of MATLAB routines for computation of the Gaussian maximum distribution, i.a. MGP. Many of these routines are available as options in the Wafo toolbox. An R-package is available

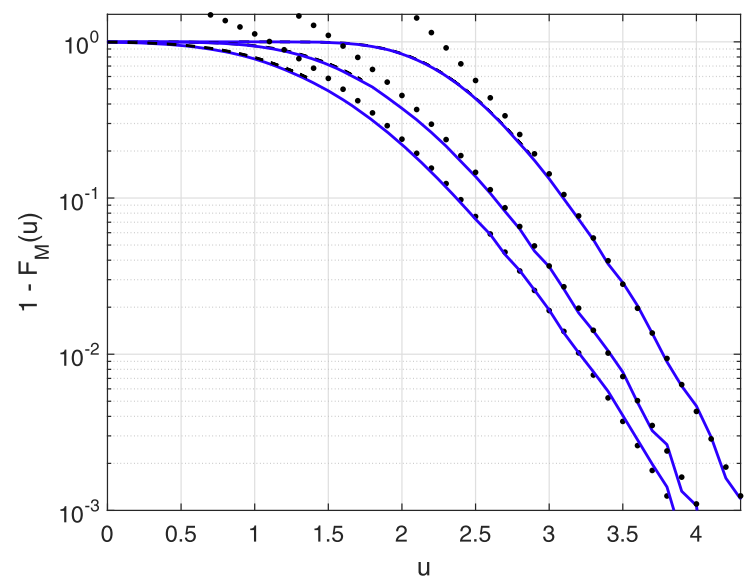

at Genz et al. (2017); see also the book by Genz and Bretz (2009).

\subsection{Examples of the "Exact" Methods}

We give examples of the use of the Rice, RIND, and MGP methods for the maximum of a stationary Gaussian process, and compare speed and accuracy. We also compute the first upcrossing time distribution, and compare with observations, generated by exact simulation from the covariance function in the Gaussian model. The MATLAB code for RIND is described in Appendix D, and that for MGP in Azaïs and Genz (2013). Both MGP and RIND can use symbolic covariance functions as arguments.

3.4.1 Maximum of Gaussian processes with "sinc" and "Gaussian" covariance. The low-frequency white noise process has constant spectral density up to a finite cut-off point and its covariance function is the "sinc" function. The standardized version, with $\mathrm{V}[X(t)]=$ $\mathrm{V}\left[X^{\prime}(t)\right]=1$, has the covariance function

$$
r_{X}(t)=\frac{\sin \sqrt{3} t}{\sqrt{3} t}
$$

Figure 2 shows the maximum distribution approximated by the Rice method and by the RIND approach, compared to the empirical distribution function from a simulation experiment. We illustrate the results for three different interval lengths, $t=10,20,80$. The figure shows the lower bound to the tail $1-F_{M_{t}}(u)$ as a solid curve. The Rice bound, which is an upper bound to the tail, is shown as dots. The empirical distribution, based of 10,000 replicates, is shown as the dashed line. The RIND approximation is very accurate.

To get an idea of the uncertainties and reproducibility we computed the approximations for two inter-

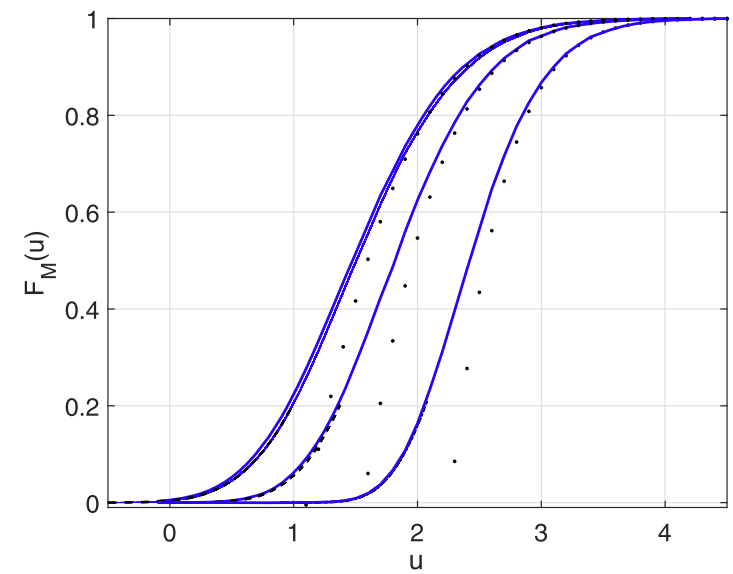

FIG. 2. Distribution of maximum over intervals of length $t=10,20,80$ for standardized low-frequency white Gaussian noise. Left: the tail of the cdf. Right: the cdf. Solid curve: the RIND result; Dots: the Rice bound; Dashed curve: the simulated cdf. 
TABLE 2

Computed and simulated exceedance probabilities for Gaussian "sinc" process, computed with MGP and RIND, together with simulated values; interval length $=2$ and 10 seconds

\begin{tabular}{lccccccc}
\hline $\boldsymbol{T = 2}$ & Levels: & $\mathbf{- 2}$ & $\mathbf{- 1}$ & $\mathbf{0}$ & $\mathbf{1}$ & $\mathbf{2}$ & $\mathbf{3}$ \\
\hline MGP & Upper & 0.9997 & 0.9821 & 0.7921 & 0.3502 & 0.0660 & 0.0049 \\
& Lower & 0.9997 & 0.9817 & 0.7895 & 0.3489 & 0.0647 & 0.0048 \\
RIND & Lower & 0.9997 & 0.9820 & 0.7916 & 0.3496 & 0.0653 & 0.0049 \\
Simulation & 4,000,000 repl & 0.9997 & 0.9819 & 0.7912 & 0.3494 & 0.0657 & 0.0049 \\
\hline $\boldsymbol{T = 1 0}$ & Levels: & $\mathbf{- 2}$ & $\mathbf{- 1}$ & $\mathbf{0}$ & $\mathbf{1}$ & $\mathbf{2}$ & $\mathbf{3}$ \\
\hline MGP & Upper & 1.0000 & 1.0002 & 0.9954 & 0.7750 & 0.2209 & 0.0189 \\
& Lower & 1.0000 & 1.0000 & 0.9944 & 0.7712 & 0.2179 & 0.0179 \\
RIND & Lower & 1.0000 & 1.0000 & 0.9947 & 0.7751 & 0.2199 & 0.0183 \\
Simulation & 4,000,000 repl & 1.0000 & 1.0000 & 0.9947 & 0.7752 & 0.2206 & 0.0190 \\
\hline
\end{tabular}

val lengths, 2, 10 seconds, and six different levels, $-2,-1, \ldots, 3$, for the Gaussian low-frequency white noise process. Table 2 shows: computed upper and lower bounds for $\mathrm{P}\left(M_{t}>u\right)$ from MGP, and computed lower bound from RIND.

As a second example we compare MGP and RIND on a Gaussian process with "Gaussian", that is, squared exponential covariance function

$$
r_{X}(t)=\exp \left(-t^{2} / 2\right),
$$

which is an example in Azaïs and Genz (2013), Example 5.1. In contrast to the oscillating "sinc" covariance, the Gaussian covariance decreases with time, and there is no typical periodicity in the sample functions. The results in Table 3 deviate slightly from those in Azaïs and Genz (2013), Table 1, since we use a denser integration grid. Both methods give good results, but RIND is the fastest. For large levels the simple Rice method is best.

Experiments with longer intervals, up to $100 \mathrm{sec}-$ onds, reveal that RIND, for some covariances, can give good results up to at least the upper one percent quantile, but for others, it underestimates the exceedance probability.

3.4.2 Time to first upcrossing. The time to first upcrossing is a "dual" to the maximum over an interval, and the normalized function $\widetilde{f}_{T_{u}}(t),(4)$, is the density of first upcrossing time for samples that start below $u$. It can be computed directly by the RIND function, as described in Appendix D.

Figure 3 shows the conditional pdf, $\widetilde{f}_{T_{u}}(t)$, for time to first upcrossing of levels $u=0,1$ for realizations starting below $u$. The histograms are based on about 650,000 realizations. The oscillating "sinc" covariance function induces small oscillations in the first crossing density for level $u=1$, and these are found by the algorithm.

\subsection{Theoretical Aspects on Rice Series for Maximum and First Upcrossing}

The Rice in- and exclusion series for the time to first upcrossing is a simplified version of the original Rice

TABLE 3

Computed and simulated exceedance probabilities with "Gaussian" covariance, computed with MGP and RIND, together with simulated values; interval length $=1$ second

\begin{tabular}{lcccccrr}
\hline $\boldsymbol{T}=\mathbf{1}$ & Levels: & $\mathbf{- 2}$ & $\mathbf{- 1}$ & $\mathbf{0}$ & $\mathbf{1}$ & $\mathbf{2}$ & $\mathbf{3}$ \\
\hline MGP & Upper & 0.9944 & 0.9279 & 0.6528 & 0.2542 & 0.0443 \\
& Lower & 0.9944 & 0.9275 & 0.6524 & 0.2540 & 0.0441 & 0.0031 \\
RIND & Lower & 0.9944 & 0.9279 & 0.6527 & 0.2543 & 0.0446 & 0.0029 \\
Simulation & $4,000,000$ repl & 0.9944 & 0.9280 & 0.6527 & 0.2543 & 0.0445 \\
\hline
\end{tabular}



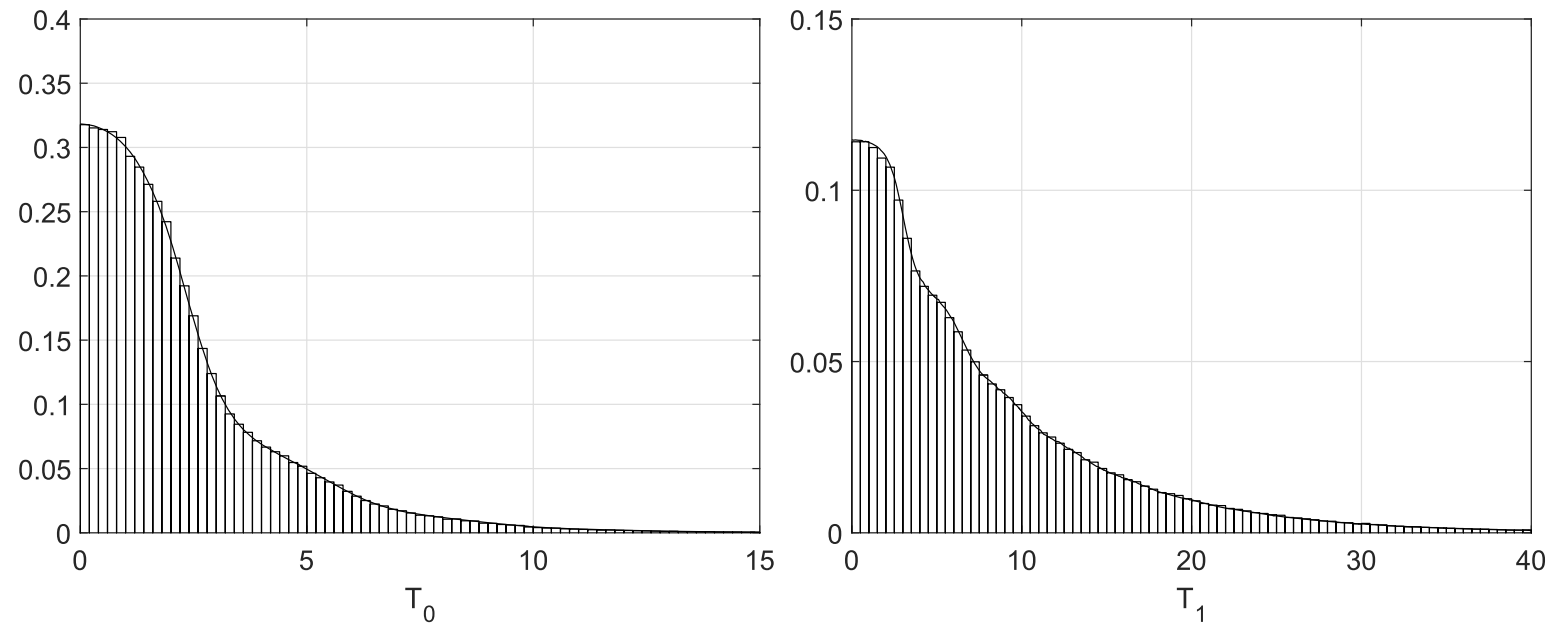

FIG. 3. Left: Pdf computed by RIND for time to first upcrossing of zero level for low-frequency Gaussian white noise, for realizations starting below zero. Right: Similar for level $u=1$. The histograms are based on about 650,000 realizations.

series for crossing intervals, (1), the difference being the starting condition: $X(0)<u$ instead of $X(0)=u$, upcrossing. Even though the formula is simple and often referred to in applications, the theoretical foundations are only partially explored, and its value as a computational tool is limited. We here refer to the investigations by Azaïs and Wschebor (2002); see also Azaiis and Wschebor (2009), Chapter 5.

Let $U_{u}=U_{u}(T, X)$ be the number of upcrossings of the level $u$ by $X(t)$ in the interval $[0, T]$, and write $U_{u}^{[m]}=U_{u}\left(U_{u}-1\right) \cdots\left(U_{u}-m+1\right)$ for the $m$ th factorial. Then,

$$
\widetilde{\alpha}_{m}=\mathrm{E}\left[U_{u}^{[m]} \mathbf{1}_{\{X(0)<u\}}\right]
$$

is the factorial moment of the number of upcrossings, counting only samples that start below $u$. We state the Rice series for $\mathrm{P}\left(M_{t}>u\right)$, and then give some details on its validity.

"The Rice series" is the expansion

$$
\begin{aligned}
1-F_{M_{t}}(u)= & \mathrm{P}(X(0) \geq u) \\
& +\sum_{m=1}^{\infty}(-1)^{m+1} \frac{\widetilde{\alpha}_{m}(t)}{m !},
\end{aligned}
$$

where, with $\boldsymbol{t}=\left(t_{1}, \ldots, t_{m}\right)$,

$$
\widetilde{\alpha}_{m}(t)=\int_{[0, t]^{m}} \widetilde{v}_{m}(\boldsymbol{t}) \mathrm{d} t_{1} \cdots \mathrm{d} t_{m}
$$

is the factorial moment, and

$$
\begin{aligned}
\widetilde{v}_{m}(\boldsymbol{t})= & \int_{x=-\infty}^{u} \mathrm{E}\left[\prod_{k=1}^{m} X^{\prime}\left(t_{k}\right)^{+} \mid\right. \\
& \left.X(0)=x, X\left(t_{1}\right)=\cdots X\left(t_{m}\right)=u\right] \\
& \cdot f_{X(0), X\left(t_{1}\right), \ldots, X\left(t_{m}\right)}(x, u, \ldots, u) \mathrm{d} x \\
= & \int_{-\infty}^{u} f_{X(0)}(x) \mathrm{E}\left[\prod_{k=1}^{m} X^{\prime}\left(t_{k}\right)^{+} \mid\right. \\
& \left.X(0)=x, X\left(t_{1}\right)=\cdots X\left(t_{m}\right)=u\right] \\
& \cdot f_{X\left(t_{1}\right), \ldots, X\left(t_{m}\right) \mid X(0)=x}(u, \ldots, u) \mathrm{d} x,
\end{aligned}
$$

the $m$ th order $u$-upcrossing intensity for sample paths starting below $u$.

The alternating Rice series offers an automatic error control if one wants to compute the maximum distribution, under the condition that the factorial moments are finite and the series converges. We quote the results by Azaïs and Wschebor (2002) for Gaussian stationary processes.

FACT 4 (Azaïs and Wschebor, 2009, Theorems 5.6 and 5.7). (a) If the covariance function $r(t)$ for the stationary Gaussian process $X(t), t \geq 0$, has a Taylor expansion at the origin that is convergent at $2 t$, and the sample paths are infinitely differentiable, then the Rice series for $\max _{[0, t]} X(s)$ converges and (5) can be used to compute the maximum distribution. 
(b) If the sample functions are sufficiently differentiable, the truncated sum

$$
\mathrm{P}(X(0)>u)+\sum_{m=1}^{K}(-1)^{m+1} \frac{\widetilde{\alpha}_{m}(t)}{m !}
$$

over- or underestimates $\mathrm{P}\left(M_{t}>u\right)$, depending on if $K$ is odd or even. (For precise conditions on the degree of differentiability, see Azaïs and Wschebor (2009).)

In (4), we encountered the (conditional) density $\tilde{f}_{T_{u}}(t)$ for the time $T_{u}$ of first upcrossing for sample paths that start below $u$, and obviously

$$
\begin{aligned}
\mathrm{P}\left(M_{t} \geq u\right)= & \mathrm{P}(X(0) \geq u)+\mathrm{P}(X(0)<u) \\
& \cdot \mathrm{P}\left(T_{u} \leq t \mid X(0)<u\right) \\
= & \mathrm{P}(X(0) \geq u)+\mathrm{P}(X(0)<u) \\
& \cdot \int_{0}^{t} \tilde{f}_{T_{u}}(s) \mathrm{d} s .
\end{aligned}
$$

The same inclusion-exclusion arguments that lead to the alternating bounds (8) will lead to bounds for the density $\widetilde{f}_{T_{u}}(t)$ in terms of the intensities (7), a fact we will use later.

\subsection{Numerical Aspects on Rice Series for Maximum and First Upcrossing}

Formula (5) contains truncated moments of derivatives in the conditional Gaussian distribution, conditioned on the process values at the selected time points, and on the starting value $X(0)=x<u$. Indeed, these moment can be expressed as functions of conditional expectations and covariances; Rosenbaum (1961) gives the explicit expression for bivariate normal variables, Kan and Robotti (2017) give an overview of recursion formulas for general dimension and a reference to efficient software. We describe how to calculate the first three terms in the Rice series by exact truncated moments. Azaiis and Wschebor (2009) refer to explicit expressions for the first two terms given in CiercoAyrolles, Croquette and Delmas (2003) but use MonteCarlo simulation for the third.

To evaluate (6) we compute the intensity, conditioned on $X(0)=x$,

$$
\widetilde{v}_{m}(\boldsymbol{t} ; x)=\mathrm{E}\left[\prod_{k=1}^{m} X^{\prime}\left(t_{k}\right)^{+} \mid\right.
$$

$$
\begin{aligned}
& X(0)=x, X\left(t_{1}\right)=\cdots X\left(t_{m}\right)=u \\
& \cdot f_{X\left(t_{1}\right), \ldots, X\left(t_{m}\right) \mid X(0)=x}(u, \ldots, u),
\end{aligned}
$$

before we integrate over $[0, t]^{m}$ and take the average over $X(0)=x<u$.

We express the first three expectations,

$$
\begin{aligned}
& \mathrm{E}\left[X^{\prime}\left(t_{1}\right)^{+} \mid X(0)=x, X\left(t_{1}\right)=u\right], \\
& \mathrm{E}\left[X^{\prime}\left(t_{1}\right)^{+} X^{\prime}\left(t_{2}\right)^{+} \mid X(0)=x,\right. \\
& \left.\quad X\left(t_{1}\right)=X\left(t_{2}\right)=u\right], \\
& \mathrm{E}\left[X^{\prime}\left(t_{1}\right)^{+} X^{\prime}\left(t_{2}\right)^{+} X^{\prime}\left(t_{3}\right)^{+} \mid X(0)=x,\right. \\
& \left.X\left(t_{1}\right)=X\left(t_{2}\right)=X\left(t_{3}\right)=u\right],
\end{aligned}
$$

as follows.

With $y^{+}=\max (0, y)$, the moments $\mathrm{E}\left(Y_{1}^{+}\right)$, $\mathrm{E}\left(Y_{1}^{+} Y_{2}^{+}\right)$, and $\mathrm{E}\left(Y_{1}^{+} Y_{2}^{+} Y_{3}^{+}\right)$for Gaussian variables can be expressed by means of functions, $\psi_{1}, \psi_{2}$, and $\psi_{3}$. The first two are

$$
\begin{aligned}
\psi_{1}(y)= & \phi(y)+y \Phi(y), \\
\psi_{2}\left(y_{1}, y_{2}, \rho\right)= & \left(y_{1} y_{2}+\rho\right) \Phi_{2}\left(y_{1}, y_{2} ; \rho\right) \\
& +y_{2} \phi\left(y_{1}\right) \Phi\left(w_{2 \cdot 1}\right) \\
& +y_{1} \phi\left(y_{2}\right) \Phi\left(w_{1 \cdot 2}\right) \\
& +\left(1-\rho^{2}\right) \phi_{2}\left(y_{1}, y_{2} ; \rho\right),
\end{aligned}
$$

where $\phi, \phi_{1}$ and $\Phi, \Phi_{2}$ are the standard uni- and bivariate, with correlation $\rho$, normal densities and distribution functions, and $w_{i \cdot j}=\left(y_{i}-\rho y_{j}\right) /\left(1-\rho^{2}\right)^{1 / 2}$. The third function, $\psi_{3}$, has a similar structure as $\psi_{2}$ but the expression is lengthy and we give it in the appendix, (21).

FACT 5. For Gaussian variables $Y_{1}, Y_{2}, Y_{3}$ with mean $\boldsymbol{m}=\left(m_{1}, m_{2}, m_{2}\right)$, variances $\left(s_{1}^{2}, s_{2}^{2}, s_{3}^{2}\right)$, covariance matrix $\boldsymbol{R}=\left(\operatorname{Cov}\left(Y_{i}, Y_{j}\right)\right)$, and correlation matrix $\boldsymbol{\rho}=\left(\operatorname{Corr}\left(Y_{i}, Y_{j}\right)\right)$,

$$
\begin{aligned}
\mathrm{E}\left(Y_{1}^{+}\right) & =\Psi_{1}\left(m_{1}, s_{1}\right):=s \psi_{1}(m / s), \\
\mathrm{E}\left(Y_{1}^{+} Y_{2}^{+}\right) & =\Psi_{2}(\boldsymbol{m}, \boldsymbol{R}) \\
& :=s_{1} s_{2} \psi_{2}\left(m_{1} / s_{1}, m_{2} / s_{2} ; \boldsymbol{\rho}\right), \\
\mathrm{E}\left(Y_{1}^{+} Y_{2}^{+} Y_{3}^{+}\right) & =\Psi_{3}(\boldsymbol{m}, \boldsymbol{R}) \\
& :=s_{1} s_{2} s_{3} \psi_{3}\left(m_{1} / s_{1}, m_{2} / s_{2}, m_{3} / s_{3} ; \boldsymbol{\rho}\right) .
\end{aligned}
$$

We express the truncated expectations in (10) in terms of the $\Psi$-functions. We need the joint normal distribution of derivatives $X^{\prime}(s)$ and process values $X(t)$, with $\operatorname{Cov}\left(X^{\prime}(s), X(t)\right)=\frac{\partial}{\partial s} r(s, t)$ and $\operatorname{Cov}\left(X^{\prime}(s)\right.$, $\left.X^{\prime}(t)\right)=\frac{\partial^{2}}{\partial s \partial t} r(s, t)$.

To simplify notation we use $\boldsymbol{m}_{2 \cdot 1}^{k}, \boldsymbol{R}_{2 \cdot 1}^{k}$ to denote the conditional mean and covariance matrix for deriva- 
tives, given process values:

$$
\begin{aligned}
\left(X^{\prime}(r), X^{\prime}(s), X^{\prime}(t)\right) \mid X(0) & =x, \\
(X(r), X(s), X(t)) & =(u, u, u) \\
& \sim N_{3}\left(\boldsymbol{m}_{2 \cdot 1}^{3}, \boldsymbol{R}_{2 \cdot 1}^{3}\right) .
\end{aligned}
$$

FACT 6. The conditional truncated moments (10) are given by the functions $\Psi_{k}\left(\boldsymbol{m}_{2 \cdot 1}^{k}, \boldsymbol{R}_{2 \cdot 1}^{k}\right)$ in (11).

Finally, the factorial moments and intensities have to be evaluated by numerical integration, for example, as

$$
\begin{aligned}
& \widetilde{\alpha}_{m}(t) \\
& =\int_{x=-\infty}^{u} f_{X(0)}(x)\left[\int_{[0, t]^{m}} \Psi_{m}\left(\boldsymbol{m}_{2 \cdot 1}^{m}, \boldsymbol{R}_{2 \cdot 1}^{m}\right)\right. \\
& \left.\quad \cdot f_{X\left(t_{1}\right), \ldots, X\left(t_{m}\right) \mid X(0)=x}(u, \ldots, u) \mathrm{d} t_{1} \ldots \mathrm{d} t_{m}\right] \mathrm{d} x .
\end{aligned}
$$

REMARK 1. Note that all normal distributions involved in (9)-(12) are conditional on $X(0)=x$. This means that mean values and covariances for the involved $X\left(t_{k}\right), X^{\prime}\left(t_{k}\right)$ are conditioned on $X(0)=x$. For example, if the unconditioned process is stationary Gaussian with mean 0 and covariance function $r(t)$,

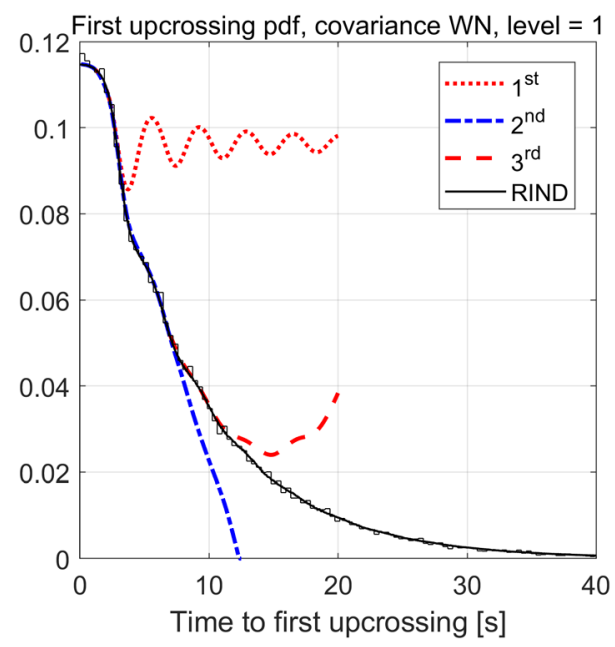

$r(0)=\lambda_{0}$,

$$
\begin{aligned}
& \mathrm{E}[X(t) \mid X(0)=x] \\
& \quad=x r(t) / \lambda_{0}, \\
& \operatorname{Cov}[X(s), X(t)] \mid X(0)=x] \\
& \quad=r(s-t)-r(s) r(t) / \lambda_{0}, \\
& \operatorname{Cov}\left[X^{\prime}(s), X^{\prime}(t) \mid X(0)=x\right] \\
& \quad=-r^{\prime \prime}(s-t)-r^{\prime}(s) r^{\prime}(t) / \lambda_{0} .
\end{aligned}
$$

\subsection{How Efficient Is the Rice Method?}

How efficient is the moment-based Rice method for maximum and first crossing, compared to the exact method, based on numerical integration? The answer depends on the character of the covariance function and on the length of the interval. We shall illustrate this on the two Gaussian processes with "sinc" and "Gaussian" covariance, and compare with the examples in Azaïs and Wschebor (2009), Chapter 5.

We use one, two, and three terms in the Rice approximation of the density for first upcrossing, and compare with simulations and with the density derived by RIND. It should be noted, however, that Azaïs and Wschebor (2009) use the unconditional $\alpha_{3}(t)$, without the initial condition $X(0)<u$, and that it is computed by simulation in their work, while we integrate to get $\widetilde{\alpha}_{3}(t)$.

Figure 4, left diagram, shows Rice approximations in Gaussian white noise for the conditional density of first upcrossing time of level $u=1$ when $X(0)<u$, and the RIND pdf $\widetilde{f}_{T_{u}}(t)$, together with histogram for simulated data. The latter two agree for the entire interval $[0,40]$. The oscillating first-order approximation

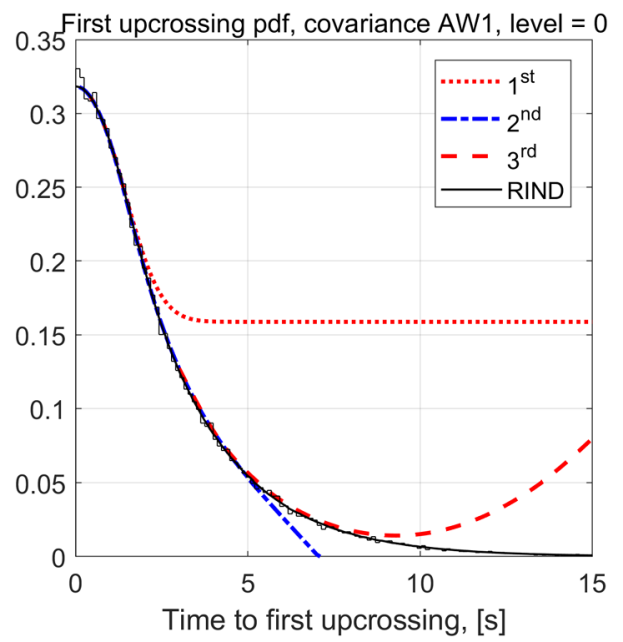

FIG. 4. Comparison between Rice approximations and exact pdffor time to first upcrossing by Gaussian process. Left: Gaussian low-frequency white noise ("sinc" covariance WN) that starts below level $u=1$. Right: Gaussian process with "Gaussian" covariance (AW-1) that starts below $u=0$. The RIND pdf agrees with simulated histogram in both cases. 
is correct for $t \leq 3$, and the second-order for $t \leq 7$, and both are computed in reasonable time. The $3 \mathrm{rd}$ order approximation takes considerable time and is correct for $t<12$. One can observe that these approximations fail to work after the respective local minima in the covariance function; see Figure 17 in Appendix A.

Figure 4, right diagram, shows the result for a Gaussian process with Gaussian covariance, AW-1 in Figure 17. Here, the Rice method gives almost correct results for time up to $t=1.5,4.5,7.5$, for the three approximations, respectively. The RIND method gives entirely correct result.

A direct comparison with Azaiis and Wschebor (2009), Table 5.1, is obtained by integrating the densities in Figure 4 to get approximations of $\mathrm{P}\left(M_{t} \geq u\right)$ for different interval lengths $t$,

$$
\begin{aligned}
\mathrm{P}\left(M_{t} \geq u\right)= & \mathrm{P}(X(0) \geq u) \\
& +\mathrm{P}(X(0)<u) \times \int_{0}^{t} \tilde{f}_{T_{u}}(s) \mathrm{d} s .
\end{aligned}
$$

The result is seen in Figure 5 for the "sinc" and "Gaussian" cases in Figure 4. As anticipated in Azaïs and Wschebor (2009), page 151, the third-order Rice approximation gives somewhat better approximation than in that work, since we use the full initial condition $X(0)<u$.

The conclusion is that the Rice approximations work for moderate interval lengths, depending on the covariance function, but that the computational cost is high. Direct integration or exact simulation from the covariance function are fast and more generally applicable.

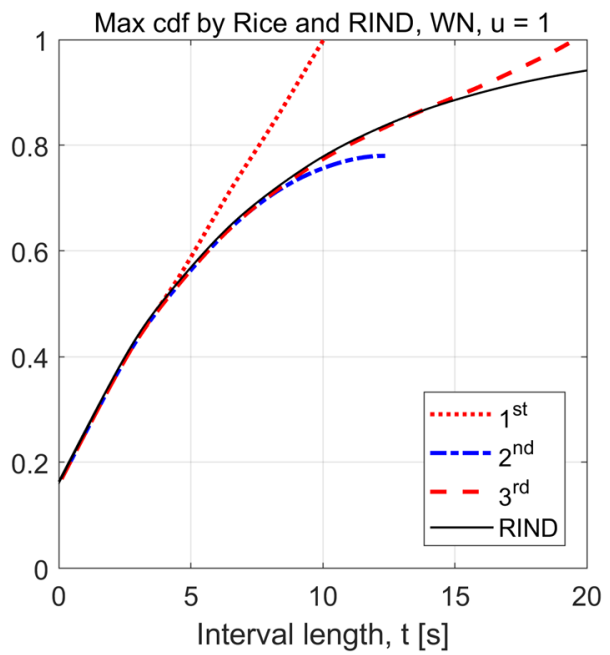

\section{LENGTH OF EXCURSIONS}

The statistical problem of zero-crossing distance in Gaussian noise was one of the main motivations behind Rice's analysis (Rice; 1944, 1945), and it was the main topic of research in the early years. Longuet-Higgins $(1962,1963)$ gave an authoritative and complete account of the knowledge at the time, comparing many alternatives to the Rice series, including new estimates of the distribution for short intervals. Wong (1966) derived the only known, nontrivial, zero crossing distribution for a differentiable Gaussian process.

Crossing intervals have remained an issue in physical and engineering applications, often generalized to length of excursions over a fixed or variable level. Estrade, Iribarren and Kratz (2012) give a theoretical overview, with many references, from a material science perspective, and discuss Rice series versus numerical integration alternatives. Most applied publications in geostatistics, material science, physics, and telecommunication use Rice series; see, for example, Smith, Hopcraft and Jakeman (2008), Brainina (2013) for the type of problems that appear. We first describe the analogy between first crossing distribution and the excursion distribution, and then we give examples for different types of covariance functions. At the end of the section we discuss the dependence between successive intervals and present a numerical exact solution.

\subsection{Comparison Between First Upcrossing and Excursion Problems}

The excursion length problem for a stationary Gaussian process has a striking similarity with the first

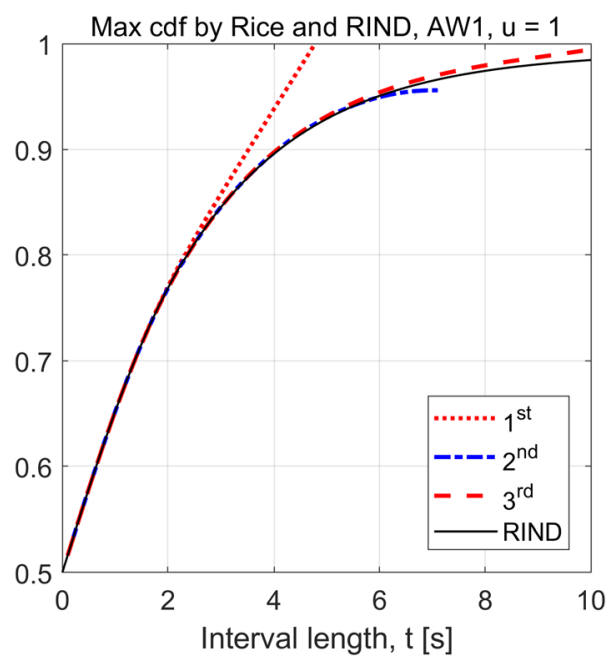

FIG. 5. Comparison between Rice approximations and exact $\mathrm{P}\left(M_{t}>u\right)$. Left: Gaussian low-frequency white noise ("sinc" covariance $W N), u=1$. Right: Gaussian process with "Gaussian" covariance $(A W-1), u=0$. The RIND curves agree with simulation in both cases. 

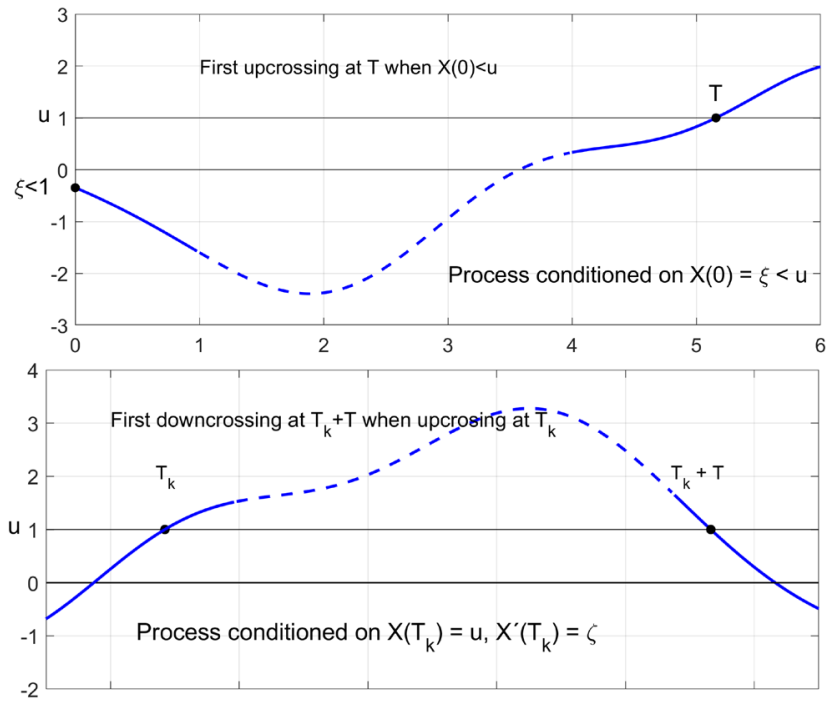

FIG. 6. Upper diagram illustrates the "first upcrossing time" for a process that starts at a random $\xi<u ; \xi$ is truncated normal. The Rice method and the exact method both put restrictions on the dashed curve. Lower diagram illustrates the "excursion length density" for a process that has an upcrossing at a time $T_{k}$, selected among all upcrossings; the random slope $\zeta$ at the upcrossing has a Rayleigh distribution (Kac and Slepian, 1959).

crossing problem in Section 3, and one can use the same Rice series and Gaussian integration routines to calculate the distributions, with just one important difference: the choice of initial condition, illustrated in Figure 6.

For the "first upcrossing density", illustrated in the upper diagram in Figure 6, we compute the intensity of an upcrossing at $T=t$, under restrictions on the process from a fixed starting point, 0 , chosen independently of the process. For the Rice series, restrictions are placed on the number of additional upcrossings, $0,1,2$, between 0 and $t$. In the exact integration method, the restriction requires that the process stays below the level in the entire interval. The curve in the diagram is a realization of a nonstationary Gaussian process with mean and covariance function (13), that starts at the randomly selected $X(0)=\xi<u$; obviously $\xi$ has an upper truncated Gaussian distribution.

For the "excursion length density", illustrated in the lower diagram, we shall compute the intensity of a downcrossing a time $T=t$ after an upcrossing at time $T_{k}$. The restrictions will be almost the same as in the "first upcrossing case", but the choice of initial point is different, it is a point of upcrossing. The distribution we seek is what we observe, in the long run, if we identify all $u$-upcrossings in an increasing time interval. Kac and Slepian (1959) proved that the em- pirical excursion distribution approaches the Rice formulation if the process is ergodic.

\subsection{Rice Bounds and Gaussian Integrals for Excursion Length Pdf}

The Rice bounds for the excursion length density are built on the $m$ th-order downcrossing intensities, writing $X(\boldsymbol{t})=\boldsymbol{u}$,

$\widetilde{v}_{m}(t)$

(14)

$$
\begin{aligned}
= & \mathrm{E}\left[X^{\prime}(0)^{+} \prod_{k=1}^{m} X^{\prime}\left(t_{k}\right)^{-} \mid X(0)=u,\right. \\
& X(\boldsymbol{t})=\boldsymbol{u}] \\
& \cdot f_{X(0), X\left(t_{1}\right), \ldots, X\left(t_{m}\right)}(u, u, \ldots, u) \mathrm{d} x
\end{aligned}
$$

$$
\begin{aligned}
= & \int_{0}^{\infty} z f_{X(0), X^{\prime}(0)}(u, z) \\
& \cdot \mathrm{E}\left[\prod_{k=1}^{m} X^{\prime}\left(t_{k}\right)^{+} \mid X(0)=u,\right. \\
& \left.X^{\prime}(0)=z, X(\boldsymbol{t})=\boldsymbol{u}\right] \\
& \cdot f_{X\left(t_{1}\right), \ldots, X\left(t_{m}\right) \mid X(0)=u, X^{\prime}(0)=z}(u, \ldots, u) \mathrm{d} z .
\end{aligned}
$$

To obtain the exact crossing intensity, we supplement (14) with the indicator

$$
\mathbf{1}_{u}^{+}(X, t)=\mathbf{1}\{X(s)>u \text { for } 0<s<t\},
$$

and get the exact density expression, when normalizing by dividing by the upcrossing intensity $v_{u}^{+}$(cf. Rychlik (1987a) and Podgórski, Rychlik and Machado (2000), equation 9),

$$
\begin{aligned}
f_{T}(t)= & \frac{1}{v_{u}^{+}} \mathrm{E}\left[\mathbf{1}_{u}^{+}(X, t) X^{\prime}(0)^{+} X^{\prime}(t)^{-} \mid\right. \\
& X(0)=X(t)=u] f_{X(0), X(t)}(u, u) \\
= & \int_{0}^{\infty} \frac{z}{\lambda_{2}} e^{-z^{2} / 2 \lambda_{2}} \mathrm{E}\left[\mathbf{1}_{u}^{+}(X, t) X^{\prime}(t)^{-} \mid\right. \\
& \left.X(0)=X(t)=u, X^{\prime}(0)=z\right] \\
& \cdot f_{X(0), X(t), X^{\prime}(0)}(u, u, z) \mathrm{d} z .
\end{aligned}
$$

The infinite-dimensional expectation in either of the expressions (15) has to be approximated by a finitedimensional one. The routine RIND, that we used in Section 3 implemented in the wave analysis package Wafo (WAFO-group, 2017a; WAFO-group, 2017b), does that to a high degree of accuracy; see Section 4.4. 


\subsection{Slepian Models}

The integration over $z$ in (15) represents an averaging over the initial random slope $\zeta$ at the start of the excursion; see Figure 6. As shown by Kac and Slepian (1959) the derivative at a generic mean level upcrossing of a stationary Gaussian process has a Rayleigh distribution, with $\lambda_{2}=\mathrm{V}[X(t)]$,

$$
f_{\zeta}(z)=\frac{z}{\lambda_{2}} e^{-z^{2} / 2 \lambda_{2}}, \quad z \geq 0 .
$$

Slepian (1963) later derived what he called "the conditional process $\widehat{x}(t)$ " for the process after a zero upcrossing. We present the model $X_{u}(t)$ after upcrossings of a general level $u$, and allow a nonstandardized variance, $\lambda_{0}=\mathrm{V}[X(t)]$.

For a stationary ergodic Gaussian process $X(t)$ with mean zero, the model for $X\left(t_{k}+t\right)$, conditioned on an upcrossing of level $u$ at $t_{k}$, is

$$
X_{u}(t)=u r(t) / \lambda_{0}-\zeta r^{\prime}(t) / \lambda_{2}+\Delta(t),
$$

where $u r(t) / \lambda_{0}-\zeta r^{\prime}(t) / \lambda_{2}$ is a regression on $X\left(t_{k}\right)=$ $u, X^{\prime}\left(t_{k}\right)=\zeta$, and $\Delta(t)$ is a nonstationary zero mean Gaussian residual process, independent of $\zeta$, with covariance function (cf. the conditional covariance in (13))

$$
C(s, t)=r(t-s)-r(s) r(t) / \lambda_{0}-r^{\prime}(s) r^{\prime}(t) / \lambda_{2} .
$$

The interpretation of the model is as the limit of the empirical distribution of the process $X\left(t_{k}+t\right)$ observed after all upcrossings $t_{k} \in[0, \infty]$, that is, "the long run distribution".

Slepian (1963) also derived a doubly conditioned model for a process with upcrossing at $t_{k}$ and downcrossing at $t_{k}+t$. Crossing conditioned models of this and similar types are named "Slepian models"; see Leadbetter, Lindgren and Rootzén (1983), Chapter 10.

It should be noted that Slepian type models can be formulated also for nonstationary Gaussian processes, even if the interpretation is not the long run sense but in Kac \& Slepian's "horizontal window" sense. Gadrich and Adler (1993) give a general treatment for crossings and local maxima. Slepian's doubly conditioned model is another example. Still other types of nonstationary Slepian models were used by Grigoriu (1989) and Lazarov and Ditlevsen (2005), in reliability applications where both the level and the process characteristics change with each occurred crossing. Abrahams (1982), inspired by Wong (1966), found an explicit expression for the crossing problem for a special type of nonstationary Gaussian process. Podgórski, Rychlik and Wallin (2015) used a Slepian model to describe vehicle movements on a non-Gaussian road. Gradient information in Gaussian processes regression is still another example (Prüher and Särkkä, 2016).

\subsection{Examples}

We give examples of crossing intervals for regular Gaussian processes with twice differentiable sample paths, and whose covariance functions have an expansion

$$
\begin{aligned}
r(t)= & 1-\lambda_{2} t^{2} / 2+\lambda_{4} t^{4} / 4 ! \\
& +o\left(|t|^{4+\epsilon}\right), \quad t \rightarrow 0 .
\end{aligned}
$$

Longuet-Higgins (1962) showed that for this type of processes, the zero-crossing distance density approaches 0 at the origin. For irregular processes, like the linear Gaussian oscillator, with a nonvanishing third-order term, $C|t|^{3} / 3$ ! in (16), the density has a nonzero limit at the origin (Longuet-Higgins, 1963).

The excursion length distribution can take many different shapes, depending on the covariance function/spectrum and on the level, and it can not be fit into any standard form. We illustrate the variety of shapes on three processes from the list in Appendix A: the low-frequency white noise (WN) process, the ocean Jonswap wave spectrum, and the rational (AW-4) spectrum from Azaïs and Wschebor (2009).

We compute Rice series of first- and second-order and "exact" distributions by means of the Wafo routine spec2tpdf for the excursion length after upcrossings of $u=-1,0,1,2$. The numerical results are compared with Monte Carlo generated excursion lengths, observed in simulated trajectories, Fourier simulated in Wafo from the spectral density. In some examples, we also compute the third-order Rice approximation. The computational cost of this is high and nothing is gained compared to the Wafo results.

Our main interest is the distribution of excursions above a nonnegative level. Upcrossings of a negative level have the same statistical properties as downcrossings of the corresponding positive level and will behave very differently from upcrossings of a positive level. As will be seen, the Rice method does not deal properly with negative levels, while the Wafo method gives good results for all covariances and levels, at a moderate or low computational cost.

The low-frequency white noise is a standard example with slowly decaying oscillating covariance function, $r(t)=\frac{\sin t}{t}$. This oscillation implies a similar oscillating density function which is most pronounced for 
excursions over low negative thresholds. The Jonswap process is the most regular of the studied processes and can be easily handled by the Wafo routine.

The processes with nonoscillating covariance are hard to handle for $u=-1$, and the second-order Rice approximation fails for $u=-1,0$ and gives good results for $u=1,2$. Wafo gives the correct distribution for all levels. When Wafo and Rice agree, the former is by far the faster routine.

4.4.1 Low-frequency white noise $(W N)$. Figure 7 and Table 5 illustrate excursion length distributions for the WN-process. Most notable is the difference between excursions above nonnegative and a negative level, which is equivalent to downcrossings of a posi- tive level. Excursions above $u=-1$ have a peak value about $t=2.5$ with a long and oscillating tail, extending beyond $T=40$. These oscillations have been the object of many experimental studies in communication theory, for example, Mimaki, Tanabe and Wolf (1981). The second-order Rice approximation ends before $t=14$, while the Wafo pdf's catch the oscillations correctly, with reasonable computing time.

Excursion pdf's for nonnegative levels are more accessible. For $u=0$ the computation time for secondorder Rice approximation is much larger than that for Wafo, and the distribution ends before $t=8$. The Wafo pdf catches the long tail correctly. For $u=1$ the second-order Rice curve agrees with the Wafo curve,
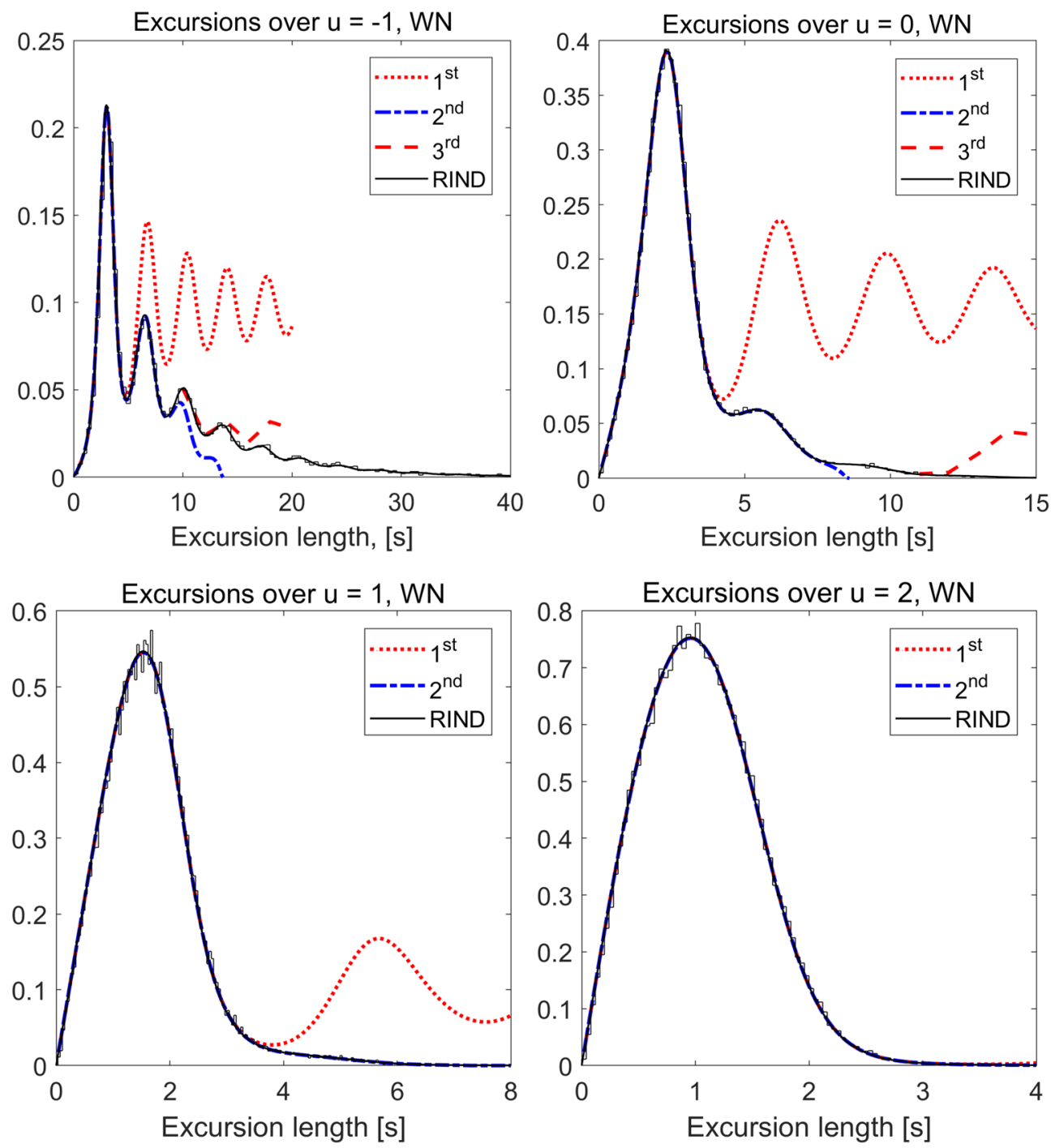

FIG. 7. Excursion length densities for low-frequency white noise: Rice series approximations (first order, dotted; second order, dash-dotted; and third order, dashed) and "exact" Wafo pdf (solid) for excursions over levels $u=-1,0,1,2$, compared with simulated histograms. Parameters from Table 5. 
and for $u=2$ even the first-order Rice curve gives the correct distribution.

For $u=0,1,2$ the time steps used for the two methods are the same; Table 5. The execution time for the Rice series is almost the same for the three levels. This is due to the nonadapting straightforward integration method used in the program. The Wafo routine spec2tpdf adapts to the complexity of the correlation function, and is very fast.

For levels $u=-1,0$ we also computed the thirdorder Rice term for a few time points to get a Rice upper limit to the density. The results are given as the dashed curve in the upper two plots in Figure 7. For $u=-1$ the improvement over the second-order approximation is small, while for $u=0$ the third-order approximation is correct up to time $t=12$. The com- putation time is very large, and the Wafo routine should be preferred.

4.4.2 Jonswap spectrum, oscillating covariance function. We use the Wafo standard Jonswap spectrum with significant waveheight $H_{s}=4 \sigma_{X}=7 \mathrm{~m}$ and peak period $T_{p}=11 \mathrm{~s}$ and normalize it to $\lambda_{0}=\lambda_{2}=1$. Its covariance function is the most oscillating one of the studied processes. The Wafo routine handles even $u=-1$ within reasonable time. The second-order Rice series ends before $t=17$ for $u=-1$ and it is also too short for $u=0$. For $u=1,2$, the second-order Rice approximation works fine, and for $u=2$ also the first order gives the correct result. Figures and data in Figure 8 and Table 6 . Note that all methods find the small bump in the pdf for $t<2$ when $u=-1$.
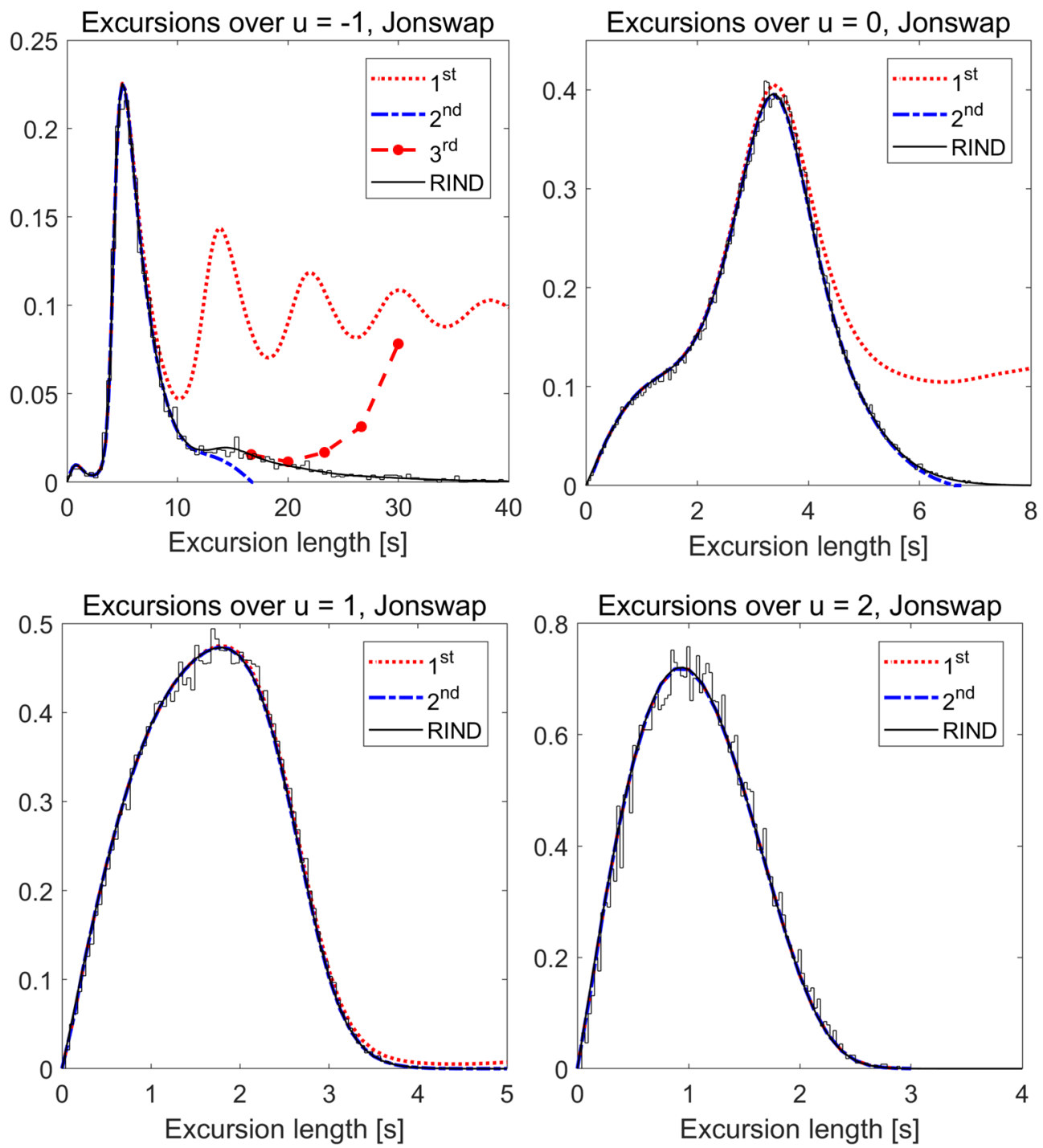

FIG. 8. Excursion length densities for Jonswap process. Parameters from Table 6. 

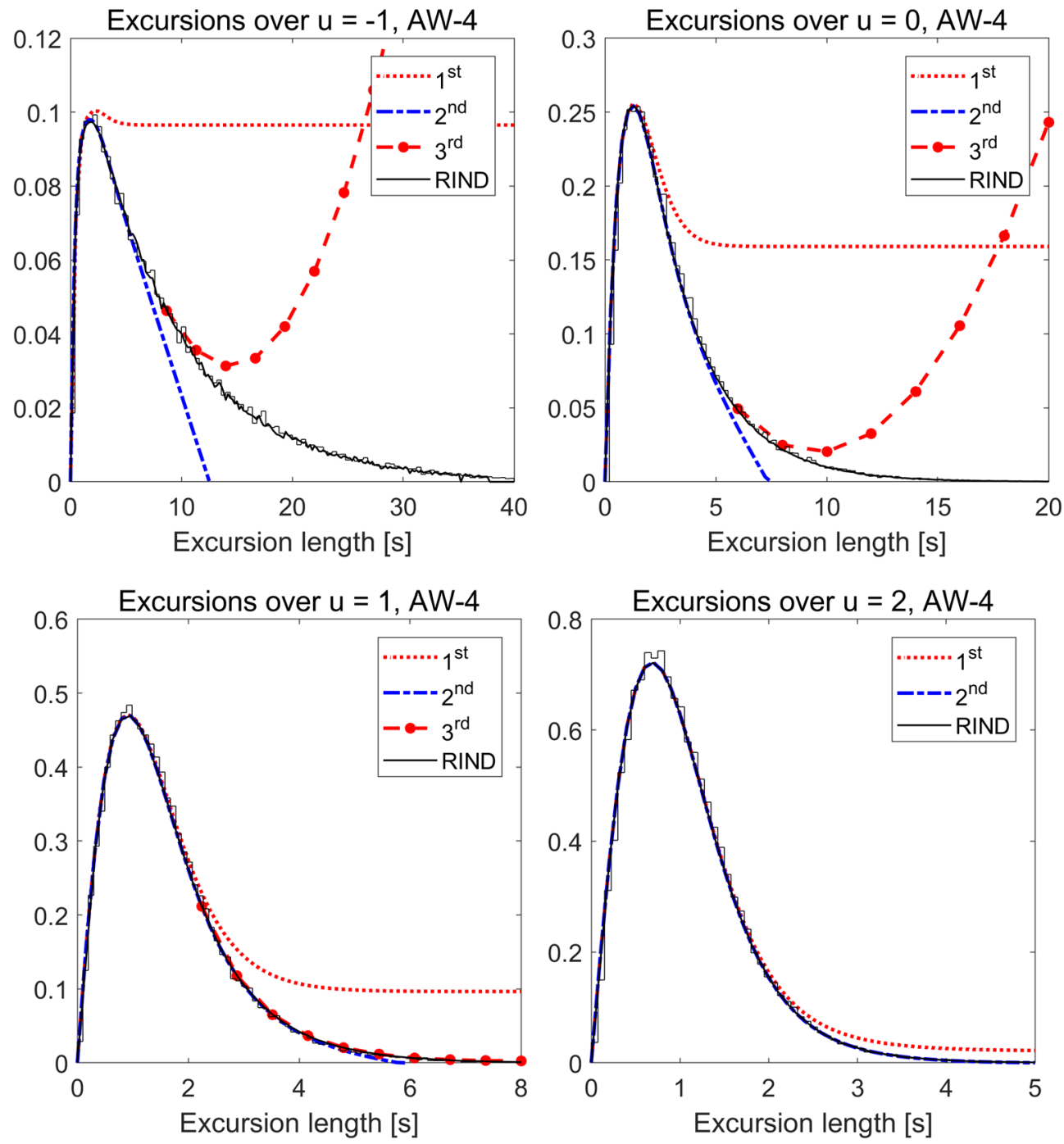

FIG. 9. Excursion length densities for covariance function AW-4. Parameters from Table 7.

4.4.3 Rational spectrum, AW-4, decreasing covariance function. The rational spectrum AW-4 is rather simple to solve with Wafo. For $u=-1,0$, the seocndorder Rice approximations end too early, before $t=12$ and $t=7$, respectively, while the Wafo pdf is correct and fast for all levels. For $u=2$ also the second-order Rice pdf is correct. The third-order series diverges quickly. Figures and data in Figure 9 and Table 7.

\subsection{Joint Distribution of Successive Zero-Crossing Distances}

The previous section dealt with the marginal distribution of individual excursion lengths. A problem of considerable practical interest is the joint distribution of successive crossing intervals, in particular the degree of dependence between crossing intervals of the mean level. We quote from Estrade, Iribarren and Kratz (2012) on a study of porous media:

So the dependence between the chordlengths (= excursions) remains an issue. Indeed, on one hand de Maré (1974) and Rychlik (1987b) proved that, in the stationary Gaussian case, the independence assumption ... fails .... On the other hand in the physics literature, the chord-length independence is generally assumed, ..., where one speaks of Independent Interval Approximation, but with no precision on the concerned probability measure.

In fact, already Longuet-Higgins (1962) proved that consecutive zero-crossing distances cannot be independent. We refer to Mimaki (1973) and Mimaki, Myoken 


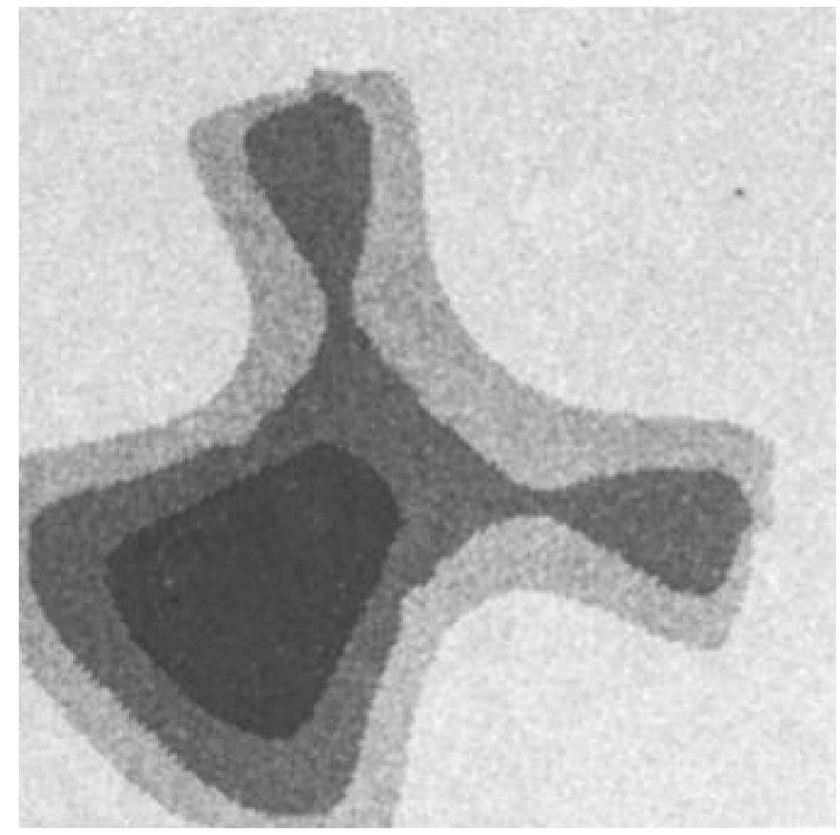

FIG. 10. Approximation of joint density for successive crossing intervals (Rychlik, 1987b).

and Kawabata (1985) for early experiments on interval dependence and its relation to spectral width, from a physical signal perspective.

The exact solution to the bivariate dependence of successive crossing intervals for a stationary Gaussian process has been known since 2000 when Podgórski, Rychlik and Machado (2000) published the Durbin type solution, and applied it in an ocean wave context. The solution has remained unnoticed outside the ocean science community. In physics literature the independence approximation has remained a popular theme even if the dependence for non-Markovian Gaussian processes is acknowledged, Sire (2008).

Wilson and Hopcraft (2017) make a systematic simulation study of the dependence for special class of correlation function, and touch upon the possibility to approximate the interval sequence by a Markov chain, an idea that goes back to McFadden (1958) and Slepian (1962). They conclude, as does McFadden, that successive intervals can be dependent even if their correlation is almost zero, and that the Markov approximation should be investigated further.

To study the Markov approximation in detail one needs the transition kernel, that is, the conditional distribution of the next interval length. We can now, by the aid of the RIND function, give an exact numerical solution to that problem. It is based on a generalization of Durbin's formula, formulated by Podgórski, Rychlik and Machado (2000), equation 10, with a formal proof in Estrade, Iribarren and Kratz (2012):

$$
\begin{aligned}
f_{T_{1}, T_{2}}\left(t_{1}, t_{2}\right) & \\
= & \frac{f_{X\left(-t_{1}\right), X(0), X\left(t_{2}\right)}(0,0,0)}{v_{0}^{+}} \\
& \cdot \mathrm{E}\left[\left\{X_{\left(-t_{1}, 0\right)}<0<X_{\left(0, t_{2}\right)}\right\}\right. \\
& \left.\cdot\left|X_{-t_{1}}^{\prime} X_{0}^{\prime} X_{t_{2}}^{\prime}\right| \mid X_{-t_{1}, 0, t_{2}}=0,0,0\right] .
\end{aligned}
$$

The density (17) for two successive zero-crossing distances, $T_{1}, T_{2}$, can be computed by RIND as described in Appendix D with moderate computing time, which gives excellent agreement with simulated data. Figure 11 shows the exact pdf for low-frequency white noise WN, Jonswap spectrum, shifted Gaussian spectrum SG-3, and the rational AW-4 spectrum. The level curves in the theoretical pdf's are chosen to include $10,30, \ldots, 99,99.9 \%$ of the distribution, and they are compared to level curves in a kernel estimated pdf, based on more than 600,000 spectral simulated waves. The pdf for the parametric Jonswap wave spectrum is similar to that obtained in Podgórski, Rychlik and Machado (2000) for an empirical ocean wave spectrum. Figure 11, upper left, should be compared with Rychlik's primitive approximation in Figure 10 for the joint pdf for low frequency white noise.

Despite the great difference between the four distributions the agreement between computed distributions and simulations is striking. It is evident that the successive zero crossing distribution is a very complex type of distribution that hardly lets itself be described in simple statistical terms. It is also evident that two successive zero crossings intervals are dependent, contrary to the Independent Interval Approximation. The correlation coefficient is 0.01 for the two broad-banded WN and AW-4 spectra, and 0.42 and 0.20 for the narrow Jonswap and SG-3, respectively.

The conditional density for any zero-crossing distance given the length of the preceding interval can be obtained from (17). The result shows clear dependence for the Jonswap spectrum, moderate dependence for the SG-3 spectrum, and almost independence for the $\mathrm{WN}$ and the AW-4 spectra.

REMARK 2. The RIND approach to successive zero crossing intervals is not limited to the bivariate distribution. The trivariate pdf is obtained similarly to (17) by adding the condition that $X_{t_{2}+t_{3}}=0$ and including $\left|X_{t_{2}+t_{3}}^{\prime}\right|\left\{X_{t_{2}, t_{2}+t_{3}}<0\right\}$ in the conditional expectation. Since the RIND function is linear in the number of variables (see Appendix D.2), this will not in- 

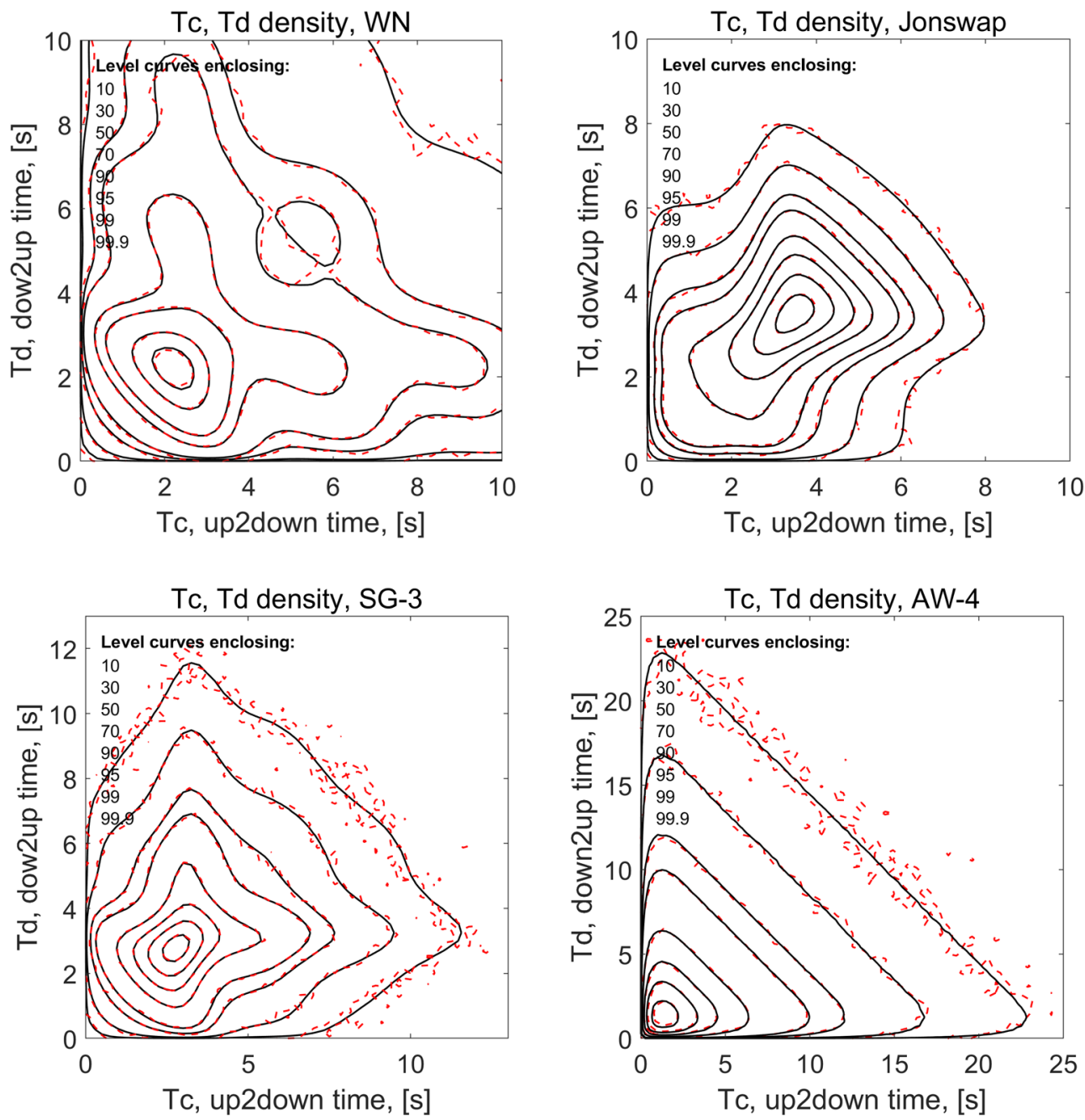

FIG. 11. Simultaneous crest/trough period pdffor low-frequency white noise (WN), Jonswap spectrum, shifted Gaussian spectrum (SG-3), and rational AW-4 spectrum, (solid) compared with simulated pdf based on more than 600,000 waves.

crease the complexity of the integration, only the computation time.

\section{MAX-MIN PERIOD AND AMPLITUDE}

\subsection{The Problem and Some of Its History}

Rice's treatises $(1944,1945)$ on random noise as a stationary Gaussian process inspired much research on stochastic models for ocean waves. StDenis and Pierson (1953) and Longuet-Higgins (1957) wrote monumental studies on the motion of ships on a "confused sea", and the statistical properties of a random surface, while Cartwright and Longuet-Higgins (1956) elaborated on Rice's results on local maxima. The Rice series type approximations for interval distributions (wave length or wave period) were studied in detail by Longuet-Higgins (1962).
Solutions were also sought to the more complex problem of joint period and amplitude distribution for individual waves, an important distribution in seakeeping studies. A partial solution for general spectral shape, based on a Rice series for an extended Slepian model (cf. Section 4.3) was given in Lindgren (1972). For narrow band spectrum an explicit expression for the joint density was derived by LonguetHiggins $(1975,1983)$. Other explicit approximations were derived by Cavanié, Arhan and Ezraty (1976) and Lindgren and Rychlik (1982). The work by Azaïs, León and Wschebor (2011) contains some interesting recent examples.

\subsection{The Exact Period/Amplitude Distribution}

In this section let us, together with Podgórski, Rychlik and Machado (2000), be inspired by the exact crossing interval density (15) in Section 4.2 to produce an 


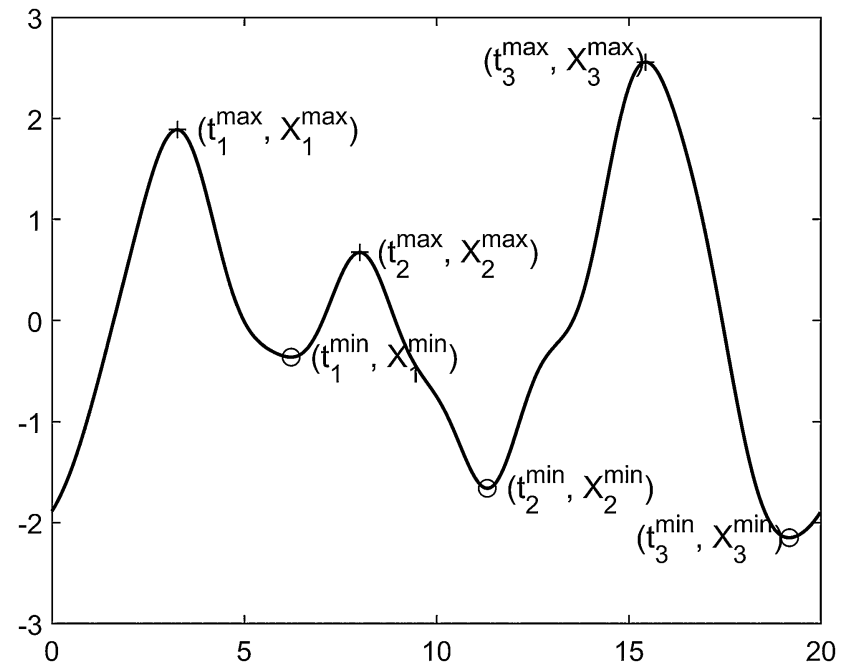

FIG. 12. Three realizations of pairs of local maxima and minima.

exact formula for the joint distribution of the height of a local maximum, the following local minimum, and the time interval in-between, here called the "half-period", illustrated in Figure 12:

$$
\begin{aligned}
X\left(t^{\mathrm{max}}\right) & =X^{\max }, \quad X\left(t^{\min }\right)=X^{\min }, \\
t^{\mathrm{max}}-t^{\mathrm{min}} & =T .
\end{aligned}
$$

The distribution is best formulated as a combination of the density of the half-period $T$ and the conditional distribution function of the maximum $=X^{\max }$ and minimum $=X^{\mathrm{min}}$, given $T=t$. Define the indicator function

$$
\begin{array}{r}
\mathbf{1}\left(t, x_{1}, x_{2}\right)=\mathbf{1}\left(X^{\prime}(s)<0,0<s<t,\right. \text { and } \\
\\
\left.X(0) \leq x_{1} \text { and } X(t) \leq x_{2}\right) .
\end{array}
$$

Then the joint distribution can be expressed as

$$
\begin{array}{r}
f_{T}(t) \times \mathrm{P}\left(X^{\max } \leq x_{1}, X^{\min } \leq x_{2} \mid T=t\right) \\
=\frac{1}{v^{\max }} \mathrm{E}\left[\mathbf{1}\left(t, x_{1}, x_{2}\right) X^{\prime \prime}(0)^{-} X^{\prime \prime}(t)^{+} \mid\right. \\
\left.X^{\prime}(0)=X^{\prime}(t)=0\right] f_{X^{\prime}(0), X^{\prime}(t)}(0,0),
\end{array}
$$

where $v^{\max }$ is the intensity of local maxima, that is, of zero downcrossings by the derivative. The conditioning, $X^{\prime}(0)=X^{\prime}(t)=0$, indicates local extremes and the second derivatives, $X^{\prime \prime}(0)^{-}, X^{\prime \prime}(t)^{+}$, specify to local maximum at 0 and minimum at $t$. The indicator qualifies the height of the extremes and the absence of further local extremes between 0 and $t$.

We will use the Wafo routine spec 2 mmtpdf (WAFO-group, 2017a), to accurately compute the three-dimensional density $f_{M, m, T}\left(x_{1}, x_{2}, t\right)$, corresponding to the distribution (18), of the height of local maximum, $M$, the following minimum, $m$, and the time $T$ in-between. The routine calls the RIND function, Appendix $\mathrm{D}$, to calculate the conditional expectations.

Integrating over $x_{1}-x_{2}=h$ one then obtains the joint amplitude, $X^{\text {max }}-X^{\text {min }}=H$, and period, $T$, distribution. Conditioning on the height of the maximum, $X^{\max }=u$, one also can see how the joint distribution of $H_{u}=u-X^{\mathrm{min}}$ and $T$ depends on $u$. More examples of cycle distributions can be found in Lindgren and Broberg (2004).

\subsection{Period and Amplitude by Rice/Slepian Type Arguments}

Lindgren (1972) used a combination of Rice type arguments and Slepian models to approximate the maxmin period and amplitude distribution for Gaussian processes with medium width spectrum. The derivation proceeds in the following steps.

Step 1: Find a second-order Rice pdf $f_{(2)}(t)$ of the time $T$ from a local maximum to the next local minimum, under the condition that the maximum has height $u$. Since $T$ is actually the length of an excursion below 0 by the process derivative the approximation $f_{(2)}(t)$ can be found by the technique in Section 4.2 applied to the derivative with the extra condition that $X(0)=u$.

Step 2: Find the joint distribution of the derivative $\zeta_{1}$ at a local maximum and $\zeta_{2}$ at a local minimum at time $\tau$ later, without any assumption of intermediate local extremes.

Step 3: Construct a Slepian model (Section 4.3),

$$
X_{u, \tau}(t)=u A_{\tau}(t)-\zeta_{1} B_{\tau, 1}(t)+\zeta_{2} B_{\tau, 2}(t)+\Delta_{\tau}(t)
$$

with regression on the height $u$ of the maximum and the derivatives at the maximum and minimum.

Step 4: Compute the distribution of $X_{u, \tau}(\tau)$ as the convolution of the normal distribution of the residual $\Delta_{\tau}(\tau)$ and the regression $u A_{\tau}(t)-\zeta_{1} B_{\tau, 1}(t)+$ $\zeta_{2} B_{\tau, 2}(t)$; see Appendix B.2 for how to do it.

\subsection{Examples}

We illustrate the exact method to compute the maxmin period and amplitude distribution and compare with the Rice method, conditioned on the maximum height.

5.4.1 Low-frequency white noise. The lowfrequency white noise has a moderately oscillating covariance function. The height-conditioned max-min amplitude and period distribution hints at a mixture of a 

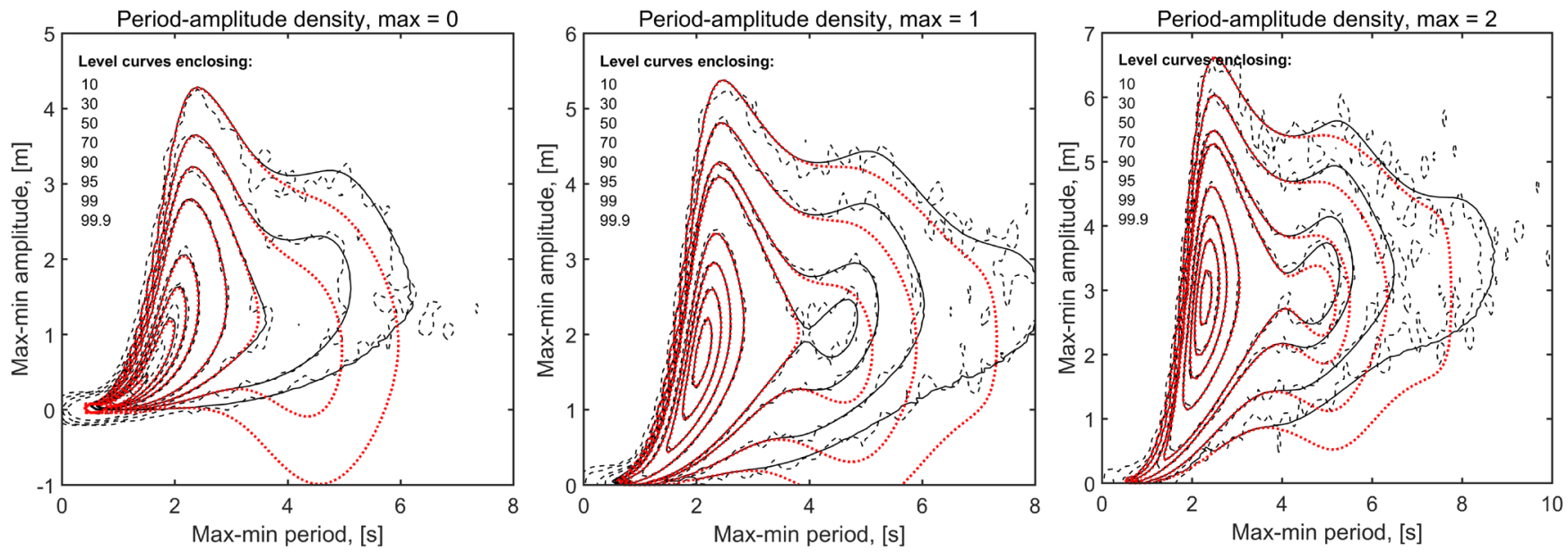

FIG. 13. Max-min period and amplitude for low-frequency white noise, conditioned on max-height $=0,1,2$. Solid $=$ Wafo $p d f$, dashed $=$ estimated pdf based on almost 100,000 period-amplitude pairs, dotted = Rice series .

majority of short waves and occasional longer waves; Figure 13. The Rice method catches the short waves but cannot identify the longer ones. Figure 14 illustrates the accuracy of the exact algorithm for the unconditioned pdf. The level curves in the theoretic density are almost identical to the empirical curves, based on almost 2.8 million cycles.

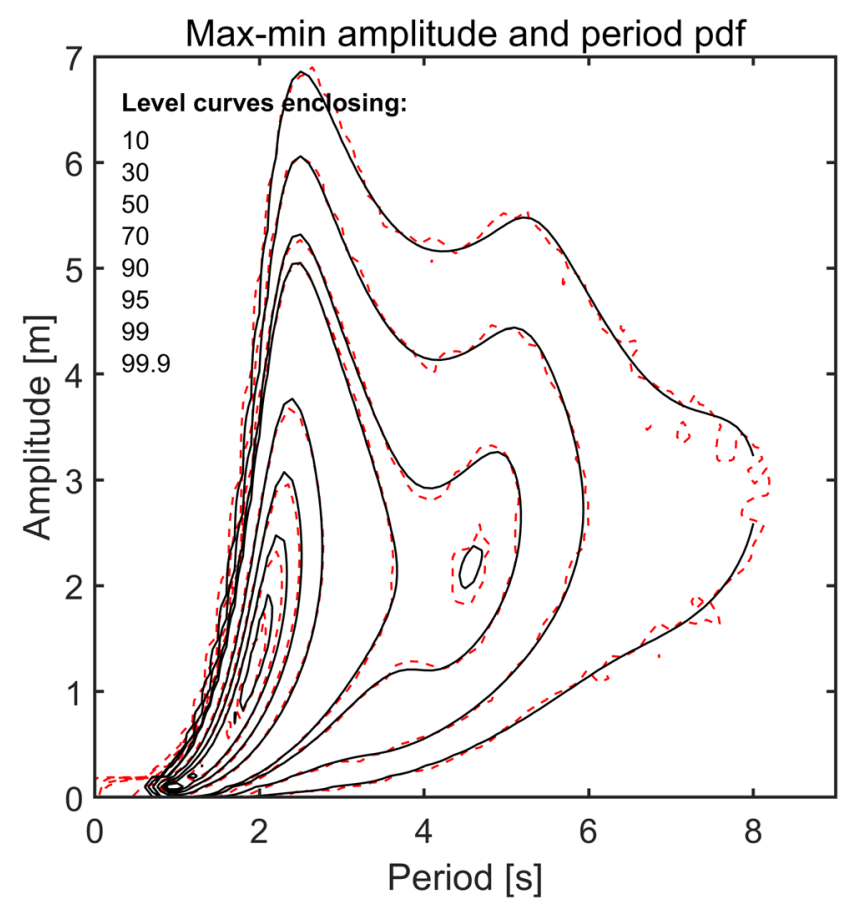

FIG. 14. Unconditional max-min amplitude and period density for low-frequency white noise compared with empirical pdf from 2.8 million waves.
5.4.2 Jonswap ocean wave spectrum. The Jonswap spectrum is a typical ocean wave spectrum and the conditional max-min amplitude and period distributions exhibit the common regular wave shape. The Rice method only catches the central part of the distribution. Figures 15-16 illustrate the excellent agreement between the Wafo computed densities and simulations.

\section{CONCLUSIONS}

Rice's formula and in- and exclusion series approximations for level crossing distributions are well known and continue to find new applications, but the scope is limited, both with respect to the type of problems that can be attacked, and to the range of model parameters. Advances in computational statistics and high-dimensional numerical quadrature since the early 1990s have made it possible to construct fast and accurate numerical routines for many more crossing related characteristics of a stationary normal process, for which only computer capacity and time define the limits.

We have compared Rice's approximations and quadrature routines for three types of problems. The distribution of the maximum of a Gaussian process over a finite interval, and the time to first curve crossing, have general statistical interest. We have studied the routines MGP, designed for the distribution of maximum, and the general purpose RIND algorithm, focusing on accuracy, computation complexity and time. Another type of problems concern the sequence of crossings and local extremes. The characteristics studied have been length of excursions over fixed levels, both the marginal distribution and the notoriously 

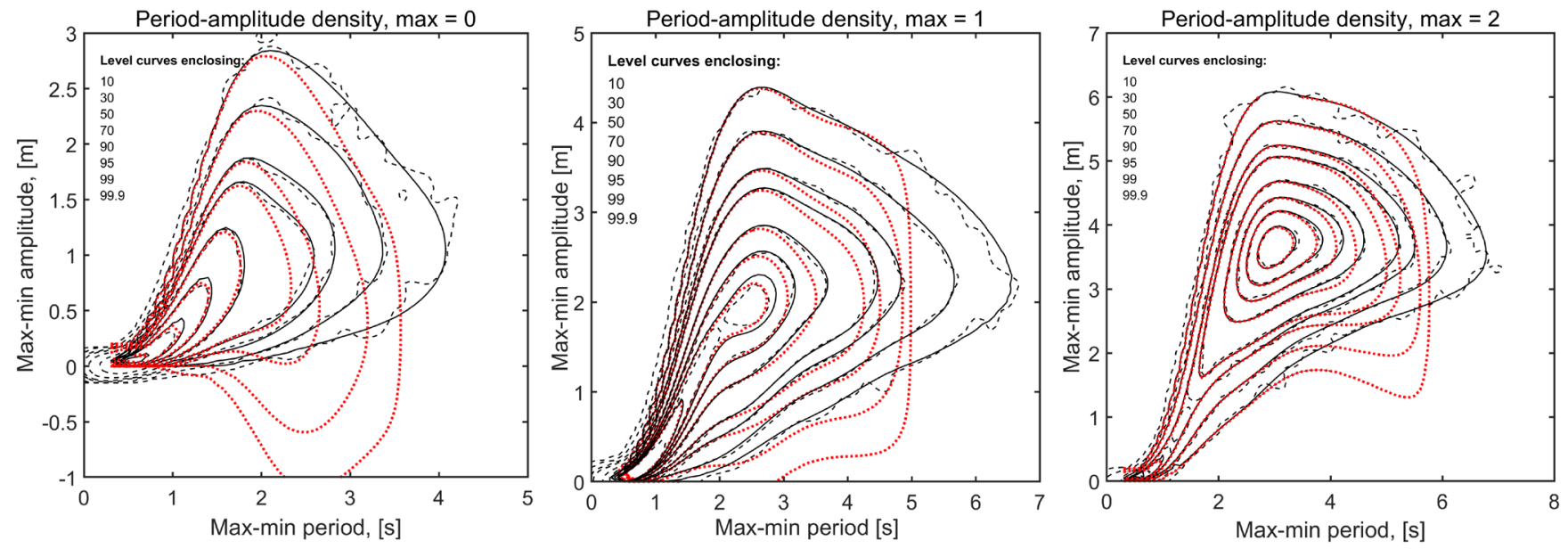

FIG. 15. Max-min period and amplitude for ocean wave spectrum Jonswap, conditioned on max-height $=0,1,2$. Solid $=$ Wafo $p d f$, dashed $=$ estimated $p d f$, dotted $=$ Rice series.

difficult dependence between successive crossing distances. The third type is the joint distribution of cycle period and amplitude.

The RIND method shows excellent agreement with simulated data for all three problem types and with reasonable, in most cases very short, execution time. The Rice method works well only for approximation of the tail of the maximum distribution, and for the length of excursions over high levels.

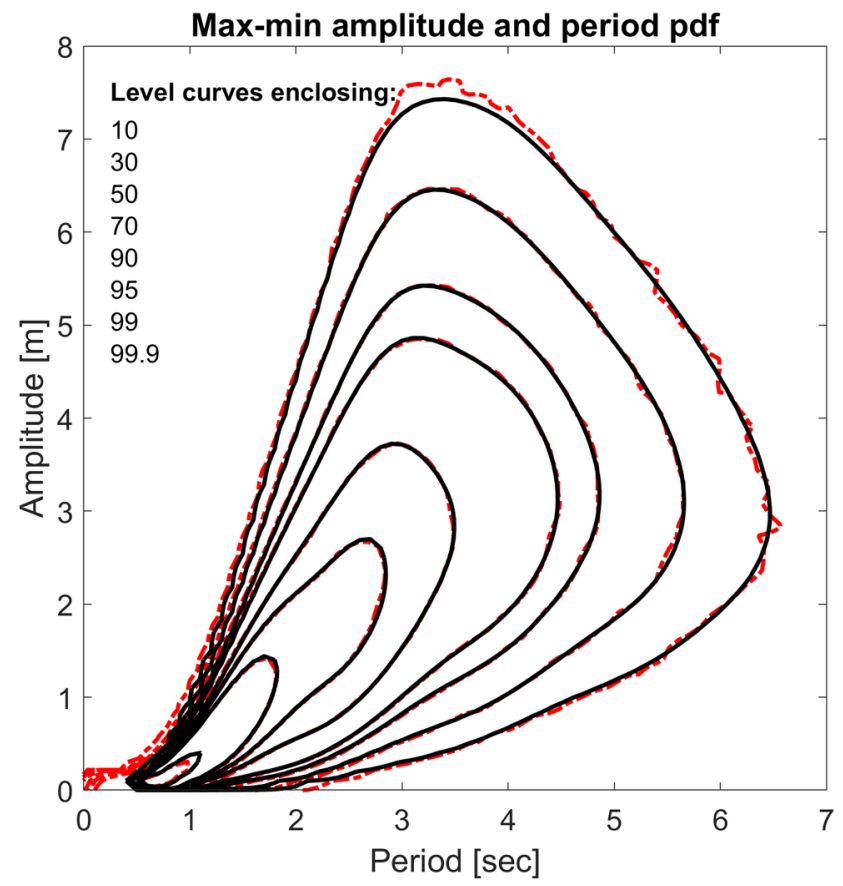

FIG. 16. Unconditional max-min amplitude and period density for Jonswap spectrum compared with empirical pdf from 2.9 million waves

\section{APPENDIX A: THE PROCESSES, COVARIANCE AND SPECTRA}

The processes in the examples have very different spectral characteristics, when normalized to have $\lambda_{0}=$ $\lambda_{2}=1$, the average length of an excursion above the mean level is $\pi$ for all the studied processes. Three processes have oscillating covariance functions and two have strictly decreasing covariance functions. The covariances AW-1, AW-4, and WN listed in Table 4 were used in Azaïs and Wschebor (2009), Chapter 5, WN also in Lindgren (1972). The shifted Gaussian spectrum SG-3 is an example of a moderately oscillating process.

The Jonswap spectrum is a realistic standard ocean wave spectrum. The explicit form is not important here; it can be found, for example, in Lindgren (2013), equation 7.33, with parameters from the WAFO-group (2017a) package.

The table gives one-sided un-normalized spectra, while the covariance functions are given in normalized form with $\lambda_{0}=\lambda_{2}=1$. The table also gives $\sqrt{\lambda_{4}}$, which for normalized spectra is equal to the mean number of local maxima per mean level upcrossing. The covariances and normalized spectra are shown in Figure 17.

\section{APPENDIX B: SOME TECHNICAL DETAILS}

\section{B.1 Truncated Multivariate Normal Moments in Section 3.6}

Let $Y_{1}, Y_{2}, Y_{3}$ be correlated normal variables with mean $\mu_{1}, \mu_{2}, \mu_{3}$, variance one, and correlation matrix $\boldsymbol{R}=\left(\operatorname{Corr}\left(Y_{i}, Y_{j}\right)\right)=\left(\rho_{i j}\right)$, and define the following 
TABLE 4

Covariance functions and spectra, ("WN" = low-frequency white noise, "SG-3" = shifted Gaussian, "AW-1" and "AW-4" spectra from Azaïs and Wschebor, 2009)

\begin{tabular}{lccr}
\hline Process & Covariance & One-sided spectrum & $\sqrt{\boldsymbol{\lambda}_{\mathbf{4}}}$ \\
\hline Nonoscillating: & $r_{1}(t)=\exp \left(-t^{2} / 2\right)$ & & 1.732 \\
"AW-1" & $r_{4}(t)=\exp (-|t| \sqrt{5}) \times P_{4}$ & $S_{1}(\omega)=\exp \left(-\omega^{2} / 2\right)$ & 2.236 \\
"AW-4" & $S_{4}(\omega)=\frac{1}{\left(\omega^{2}+5\right)^{4}}$ & \\
& $P_{4}=1+\sqrt{5}|t|+2 t^{2}+(\sqrt{5} / 3)|t|^{3}$ & & 1.342 \\
Oscillating: & $r_{w n}(t)=\frac{\sin \sqrt{3} t}{\sqrt{3} t}$ & $S_{w n}(\omega)=\mathbf{1}\{0<\omega<\sqrt{3}\}$ & 1.428 \\
"WN" & $r_{J}(t)$ & $S_{J}(\omega)=\mathrm{Jonswap}(\omega)$ & 1.38 \\
"Jonswap" & $r_{s g}(t)=\cos \left(\frac{3 t}{\sqrt{10}}\right) \exp \left(-t^{2} / 20\right)$ & $S_{s g}(\omega)=\sqrt{\pi / 5}\left(e^{-5\left(\omega-\omega_{0}\right)^{2}}+e^{-5\left(\omega+\omega_{0}\right)^{2}}\right)$ & \\
"SG-3" & & & \\
\hline
\end{tabular}

functions (Kan and Robotti, 2017), to be used for truncated moments:

$$
\begin{aligned}
\psi_{1}(y)= & \phi(y)+y \Phi(y) \\
\psi_{2}\left(y_{1}, y_{2}, \rho_{12}\right)= & \left(y_{1} y_{2}+\rho_{12}\right) \Phi_{2}\left(y_{1}, y_{2} ; \rho_{12}\right) \\
& +y_{2} \phi\left(y_{1}\right) \Phi\left(w_{2 \cdot 1}\right) \\
& +y_{1} \phi\left(y_{2}\right) \Phi\left(w_{1 \cdot 2}\right) \\
& +\left(1-\rho_{12}^{2}\right) \phi_{2}\left(y_{1}, y_{2} ; \rho_{12}\right)
\end{aligned}
$$
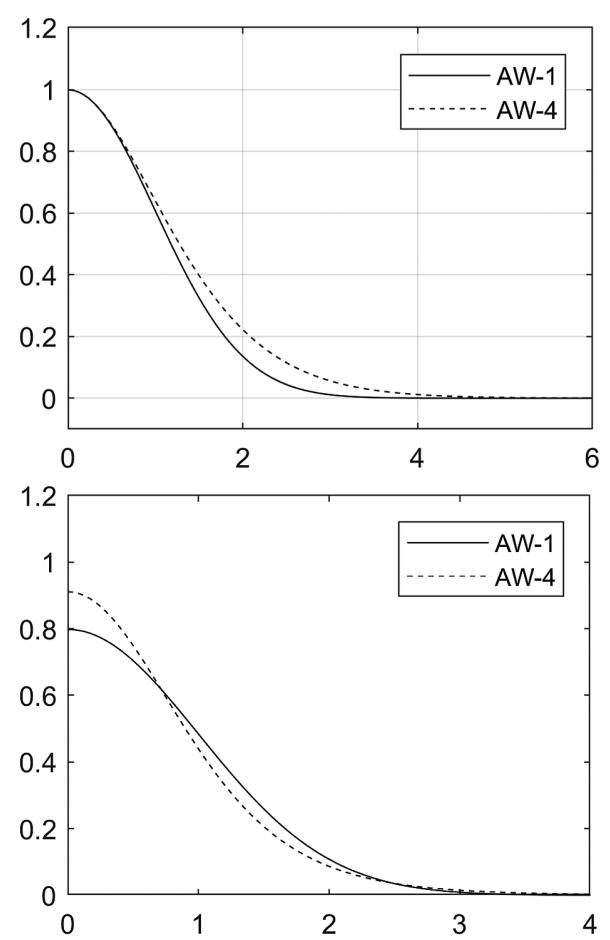

where $w_{i \cdot j}=\left(y_{i}-\rho_{i j} y_{j}\right) /\left(1-\rho_{i j}^{2}\right)^{1 / 2}$, and

$$
\begin{aligned}
\psi_{3}\left(y_{1}, y_{2}, y_{3} ; \boldsymbol{R}\right) \\
=\left(\mu_{1} \mu_{2} \mu_{3}+\rho_{23} \mu_{1}+\rho_{13} \mu_{2}+\rho_{12} \mu_{3}\right) \\
\quad . \Phi_{3}(\boldsymbol{\mu} ; \boldsymbol{R}) \\
\quad+\left(\mu_{2} \mu_{3}+\rho_{12} \rho_{13}+\rho_{23}\right) \phi\left(\mu_{1}\right) \\
\quad . \Phi_{2}\left(w_{2 \cdot 1}, w_{3 \cdot 1} ; \rho_{23 \cdot 1}\right) \\
\quad+\left(\mu_{1} \mu_{3}+\rho_{12} \rho_{23}+\rho_{13}\right) \phi\left(\mu_{2}\right)
\end{aligned}
$$
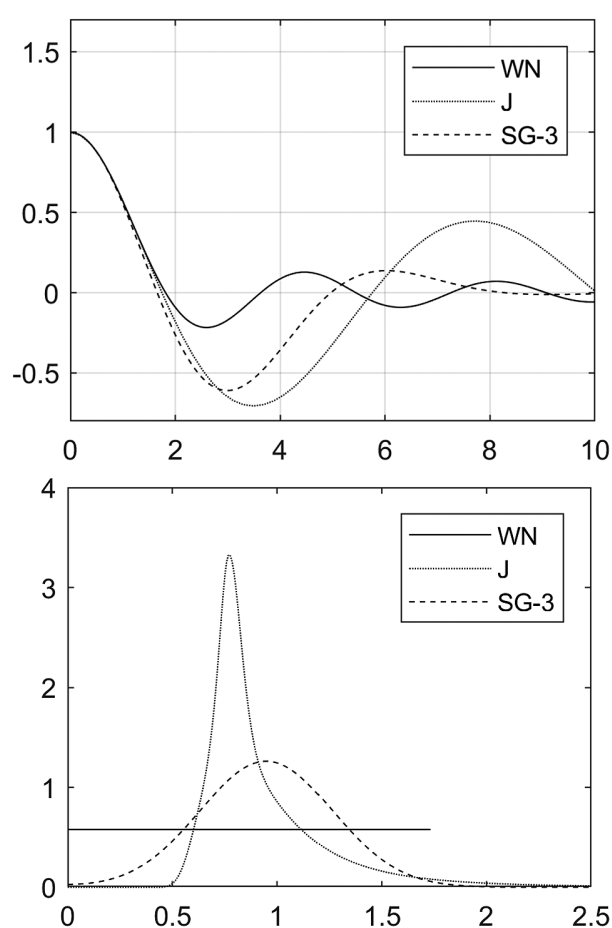

FIG. 17. Top row: Nonoscillating and oscillating covariance functions. Bottom row: Normalized spectra for nonoscillating and oscillating covariance functions. 
(21)

$$
\begin{aligned}
& \cdot \Phi_{2}\left(w_{1 \cdot 2}, w_{3 \cdot 2} ; \rho_{13 \cdot 2}\right) \\
& +\left(\mu_{1} \mu_{2}+\rho_{13} \rho_{23}+\rho_{12}\right) \phi\left(\mu_{3}\right) \\
& \cdot \Phi_{2}\left(w_{1 \cdot 3}, w_{2 \cdot 3} ; \rho_{12 \cdot 3}\right) \\
& +\left(1-\rho_{12}^{2}\right) \mu_{3} \phi_{2}\left(\mu_{1}, \mu_{2} ; \rho_{12}\right) \Phi\left(w_{3 \cdot 12}\right) \\
& +\left(1-\rho_{13}^{2}\right) \mu_{2} \phi_{2}\left(\mu_{1}, \mu_{3} ; \rho_{13}\right) \Phi\left(w_{2 \cdot 13}\right) \\
& +\left(1-\rho_{23}^{2}\right) \mu_{1} \phi_{2}\left(\mu_{2}, \mu_{3} ; \rho_{23}\right) \Phi\left(w_{1 \cdot 23}\right) \\
& +\operatorname{det}(\boldsymbol{R}) \phi_{3}(\boldsymbol{\mu} ; \boldsymbol{R}),
\end{aligned}
$$

where

$$
\begin{aligned}
& w_{i \cdot j k}=\left(w_{i \cdot k}-\rho_{i j \cdot k} w_{j \cdot k}\right) /\left(1-\rho_{i j \cdot k}^{2}\right)^{1 / 2}, \\
& \rho_{i j \cdot k}=\left(\rho_{i j}-\rho_{i k} \rho_{j k}\right) / \sqrt{\left(1-\rho_{i k}^{2}\right)\left(1-\rho_{j k}^{2}\right)} .
\end{aligned}
$$

\section{B.2 The Distribution of $\zeta_{1} B_{1}-\zeta_{2} B_{2}$ in Step 4, Page 119}

The density of $\zeta_{1} B_{1}-\zeta_{2} B_{2}$ can be expressed explicitly in terms of the function $\psi_{1}$, (19). The density can be expressed as (for simplicity, $\boldsymbol{m}=\boldsymbol{m}_{\tau}, \boldsymbol{T}=\boldsymbol{T}_{2 \cdot 1}$ ),

$$
c z_{1} z_{2} \phi_{2}(z ; \boldsymbol{m}, \boldsymbol{T}), \quad z_{1}, z_{2}>0,
$$

with $c$ a generic constant. Normalizing $\left(a_{1}, a_{2}\right)=$ $\left(B_{1},-B_{2}\right) /|B|$, we get the density by integrating over the first quadrant $\mathbf{1}$ :

$$
\begin{aligned}
& f_{\zeta_{1} B_{\tau, 1}(\tau)-\zeta_{2} B_{\tau, 2}(\tau)}(y) \\
& \quad=c \int_{\mathbf{1} \cap\left\{a_{1} z_{1}+a_{2} z_{2}=y /|B|\right\}} z_{1} z_{2} \phi_{2}(z ; \boldsymbol{m}, \boldsymbol{T}) \mathrm{d} z .
\end{aligned}
$$

An orthogonal change of integration, $\boldsymbol{v}=\boldsymbol{A} \boldsymbol{z}=$ $\left(\begin{array}{cc}a_{1} & a_{2} \\ -a_{2} & a_{1}\end{array}\right) z, z=A^{\top} \boldsymbol{v}$, with corresponding new random variable, $v=A \zeta$ with mean $\boldsymbol{n}=\boldsymbol{A m}$ and covariance matrix $\boldsymbol{C}=\boldsymbol{A} \boldsymbol{T} \boldsymbol{A}^{\top}$, brings the integral (22) on the form

$$
\begin{aligned}
f_{\zeta_{1} B_{\tau, 1}(\tau)-\zeta_{2} B_{\tau, 2}(\tau)}(y) & \\
= & c \int_{\boldsymbol{A} \mathbf{1} \cap\left\{v_{1}=y /|B|\right\}}\left(a_{1} v_{1}-a_{2} v_{2}\right)\left(a_{2} v_{1}+a_{1} v_{2}\right) \\
& \cdot \phi_{2}(\boldsymbol{v} ; \boldsymbol{n}, \boldsymbol{C}) \mathrm{d} \boldsymbol{v} \\
= & c \int_{\boldsymbol{A} \mathbf{1} \cap\left\{v_{1}=y /|B|\right\}}\left(b_{2} y^{2}+b_{1} y v_{2}-b_{0} v_{2}^{2}\right) \\
& \cdot \phi_{2}(\boldsymbol{v} ; \boldsymbol{n}, \boldsymbol{C}) \mathrm{d} \boldsymbol{v},
\end{aligned}
$$

where we define $b_{2}=a_{1} a_{2} /|B|^{2}, b_{1}=\left(a_{1}^{2}-a_{2}^{2}\right) /|B|$, $b_{0}=a_{1} a_{2}$.

In the integral (23) we can replace the twodimensional normal density for $\zeta$ by the one- dimensional conditional normal density for $\nu_{2}$ given $v_{1}=y /|B|$. It has mean and variance

$$
\begin{aligned}
n_{2 \cdot 1} & =\mathrm{E}\left(v_{2}\left|v_{1}=y /\right| B \mid\right) \\
& =n_{2}+\boldsymbol{C}_{21}\left(y /|B|-n_{1}\right) / \boldsymbol{C}_{11}, \\
\sigma_{2 \cdot 1}^{2} & =\mathrm{V}\left(v_{2}\left|v_{1}=y /\right| B \mid\right)=\boldsymbol{C}_{22}-\boldsymbol{C}_{21}^{2} / \boldsymbol{C}_{11} .
\end{aligned}
$$

Thus, the density (23) is equal to the normalized sum of three moments in a normal distribution, truncated to the interval $J=\left\{v_{2} ;\left(y /|B|, v_{2}\right) \in \boldsymbol{A} \mathbf{1}\right\}=\left(j_{1}, j_{2}\right)$, $j_{1}<j_{2}$,

$$
\begin{aligned}
& f_{\zeta_{1} B_{\tau, 1}(\tau)-\zeta_{2} B_{\tau, 2}(\tau)}(y) \\
& =c \int_{J}\left(b_{2} y^{2}+b_{1} y v_{2}-b_{0} v_{2}^{2}\right) \\
& \cdot \phi_{1}\left(v_{2} ; m_{2 \cdot 1}, \sigma_{2 \cdot 1}^{2}\right) \mathrm{d} v_{2} .
\end{aligned}
$$

For easy reference we give the standard recursion for the truncated moments for a $N\left(\mu, \sigma^{2}\right)$-distribution (Kan and Robotti, 2017), with $\alpha=\left(j_{1}-\mu\right) / \sigma, \beta=$ $\left(j_{2}-\mu\right) / \sigma$ :

$$
\begin{aligned}
& \int_{J} \phi_{1}\left(v ; \mu, \sigma^{2}\right) \mathrm{d} v \\
& \quad=F_{0}^{1}=\Phi(\beta)-\Phi(\alpha), \\
& \int_{J} v \phi_{1}\left(v ; \mu, \sigma^{2}\right) \mathrm{d} v \\
& \quad=F_{1}^{1} \\
& \quad=\sigma\{\mu(\Phi(\beta)-\Phi(\alpha))-\sigma(\phi(\beta)-\phi(\alpha))\}, \\
& \int_{J} v^{2} \phi_{1}\left(v ; \mu, \sigma^{2}\right) \mathrm{d} v \\
& \quad=F_{2}^{1} \\
& =m F_{1}^{1}+\sigma^{2} F_{0}^{1}-\sigma\left(j_{2} \phi(\beta)-j_{1} \phi(\alpha)\right) .
\end{aligned}
$$

\section{APPENDIX C: DATA FOR EXAMPLES IN SECTION 4.4}

Tables 5-7 show interval lengths $T_{\max }$ and time steps $d t$, optional parameters for RIND and execution times for the system used for the different computations. The RIND parameter NIT defines which integration routine that is used, and the parameter SPEED sets the error limits for the integration; SPEED $=1$ is slowest and most accurate, SPEED $=9$ is the fastest. For each spectrum, Rice series and RIND were compared on the same computing system, but different systems were used for the three spectra, so only relative comparisons can be made. 
TABLE 5

Data for excursion length in low-frequency white noise $(W N)$ example

\begin{tabular}{|c|c|c|c|c|c|c|c|c|}
\hline \multirow[b]{2}{*}{$u$} & \multicolumn{3}{|c|}{ Rice series } & \multicolumn{5}{|c|}{ RIND } \\
\hline & $T_{\max }$ & $d t$ & Time (s) & $T_{\max }$ & $d t$ & NIT & SPEED & Time (s) \\
\hline-1 & 14 & 0.1 & 30 & 40 & 0.1 & -2 & 2 & 69 \\
\hline 0 & 9 & 0.05 & 65 & 15 & 0.05 & -2 & 2 & 10 \\
\hline 1 & 8 & 0.04 & 57 & 8 & 0.04 & 5 & 5 & 0.88 \\
\hline 2 & 4 & 0.02 & 59 & 4 & 0.02 & 5 & 5 & 0.18 \\
\hline
\end{tabular}

\section{APPENDIX D: THE RIND FUNCTION}

\section{D.1 The Function}

The structure of the restricted first upcrossing intensity (3),

$$
\mathrm{E}\left[\mathbf{1}_{u}(X, t) X^{\prime}(t)^{+} \mid X(t)=u\right] f_{X(t)}(u),
$$

is recurrent in all crossing problems. It contains a "crossing condition", $X(t)=u$, with density $f_{X(t)}(u)$, for the crossed level, a "bias factor", $X^{\prime}(t)^{+}$, taking care of the over-representation of large gradients at the upcrossing, and a "qualifying factor",

$$
\mathbf{1}_{u}(X, t)=\mathbf{1}\{X(s) \leq u \text { for } 0 \leq s \leq t\},
$$

for what other conditions need to be satisfied.

For other crossing intensities the precise form of the factors will be different, but the structure will be the same. For example, in (17),

$$
\begin{aligned}
& \mathrm{E}\left[\left\{X_{\left(-t_{1}, 0\right.}<0<X_{\left(0, t_{2}\right)}\right\}\left|X_{-t_{1}}^{\prime} X_{0}^{\prime} X_{t_{2}}^{\prime}\right| \mid X_{-t_{1}, 0, t_{2}}\right. \\
& \quad=0,0,0] f_{X_{-t_{1}, 0, t_{2}}}(0,0,0) .
\end{aligned}
$$

Since the indicator functions define infinitedimensional events, the conditional expectations have to be found by a numerical algorithm, approximating by finite-dimensional ones.

The RIND function is a MATLAB interface to a set of algorithms, originally written in Fortran and $\mathrm{C}++$, for computation of complex expectations like (24) in high-dimensional normal distributions. Based on ideas in Rychlik (1987c), further presented in Lindgren and Rychlik (1991), Rychlik (1992b), Podgórski, Rychlik and Machado (2000) designed an early version of the basic RIND algorithm to systematically extend the finite-dimensional distributions to obtain an accurate value of the infinite-dimensional integral.

Brodtkorb (2006) combined the RIND algorithm with numerical integration methods developed by Genz (1992, 1993), Genz and Kwong (2000), and others to an efficient and versatile routine. It is now available in the wave analysis Wafo project (WAFO-group, 2017a, 2017b), and it is also included in the extreme value package MAGP by Mercadier (2006a). The Wafo project comes both in a MATLAB version (WAFOgroup, 2017a, 2017b), and in a Python version (WAFOgroup, 2017b).

The RIND algorithm takes as input means and covariances for three groups of multivariate normal variables. One group consists of the variables to condition on, $\mathrm{Xc}=\mathrm{xc}$, a second group are the derivatives $\mathrm{Xd}$ that define the bias factors, and a third group Xt contains the variables that have to satisfy an interval condition like $\mathrm{xlo} \leq \mathrm{Xt} \leq \mathrm{xup}$. Such constraints may also be imposed on the derivatives. The mean vector and joint covariance matrix for the three groups have to be specified;

\begin{tabular}{|c|c|c|c|c|c|c|c|c|}
\hline \multirow[b]{2}{*}{$u$} & \multicolumn{3}{|c|}{ Rice series } & \multicolumn{5}{|c|}{ RIND } \\
\hline & $T_{\max }$ & $d t$ & Time (s) & $T_{\max }$ & $d t$ & NIT & SPEED & Time (s) \\
\hline-1 & 17 & 0.2 & 8.8 & 40 & 0.25 & -2 & 2 & 51 \\
\hline 0 & 7 & 0.1 & 8.3 & 8 & 0.05 & -2 & 2 & 6.2 \\
\hline 1 & 5 & 0.1 & 4.7 & 5 & 0.1 & 7 & 5 & 0.16 \\
\hline 2 & 4 & 0.05 & 7.7 & 4 & 0.05 & 7 & 5 & 0.15 \\
\hline
\end{tabular}
see the help text for the routine in the Wafo package.

TABLE 6

Data for excursion length in Jonswap example 
TABLE 7

Data for excursion length in AW-4 example

\begin{tabular}{|c|c|c|c|c|c|c|c|c|}
\hline \multirow[b]{2}{*}{$u$} & \multicolumn{3}{|c|}{ Rice series } & \multicolumn{5}{|c|}{ RIND } \\
\hline & $T_{\max }$ & $d t$ & Time (s) & $T_{\max }$ & $d t$ & NIT & SPEED & Time (s) \\
\hline-1 & 15 & 0.1 & 40 & 40 & 0.2 & -2 & 9 & 3.0 \\
\hline 0 & 8 & 0.25 & 1.8 & 20 & 0.1 & -2 & 5 & 4.5 \\
\hline 1 & 8 & 0.16 & 4.7 & 8 & 0.16 & -2 & 5 & 1.2 \\
\hline 2 & 5 & 0.1 & 4.7 & 5 & 0.1 & -2 & 5 & 1.2 \\
\hline
\end{tabular}

In Section 3 we used RIND directly to compute maximum and crossing interval distributions. Another way to use RIND is to embed it in a problem specific code, depending of the type of crossing distribution. Examples of specific crossing routines in the Wafo package in MATLAB are:

spec2tpdf: computes exceedance length density for general level,

spec2ttpdf: computes joint density of successive zero-crossing distances, spec2thpdf: computes joint density of crest period and amplitude of wave,

spec2mmtpdf: computes different three-dimensional distributions related to local maxima and minima,

spec2tapdf: computes conditional and unconditional max-min amplitude and period pdf (based on spec2mmtpdf).

\section{D.2 Calling RIND in Matlab Wafo}

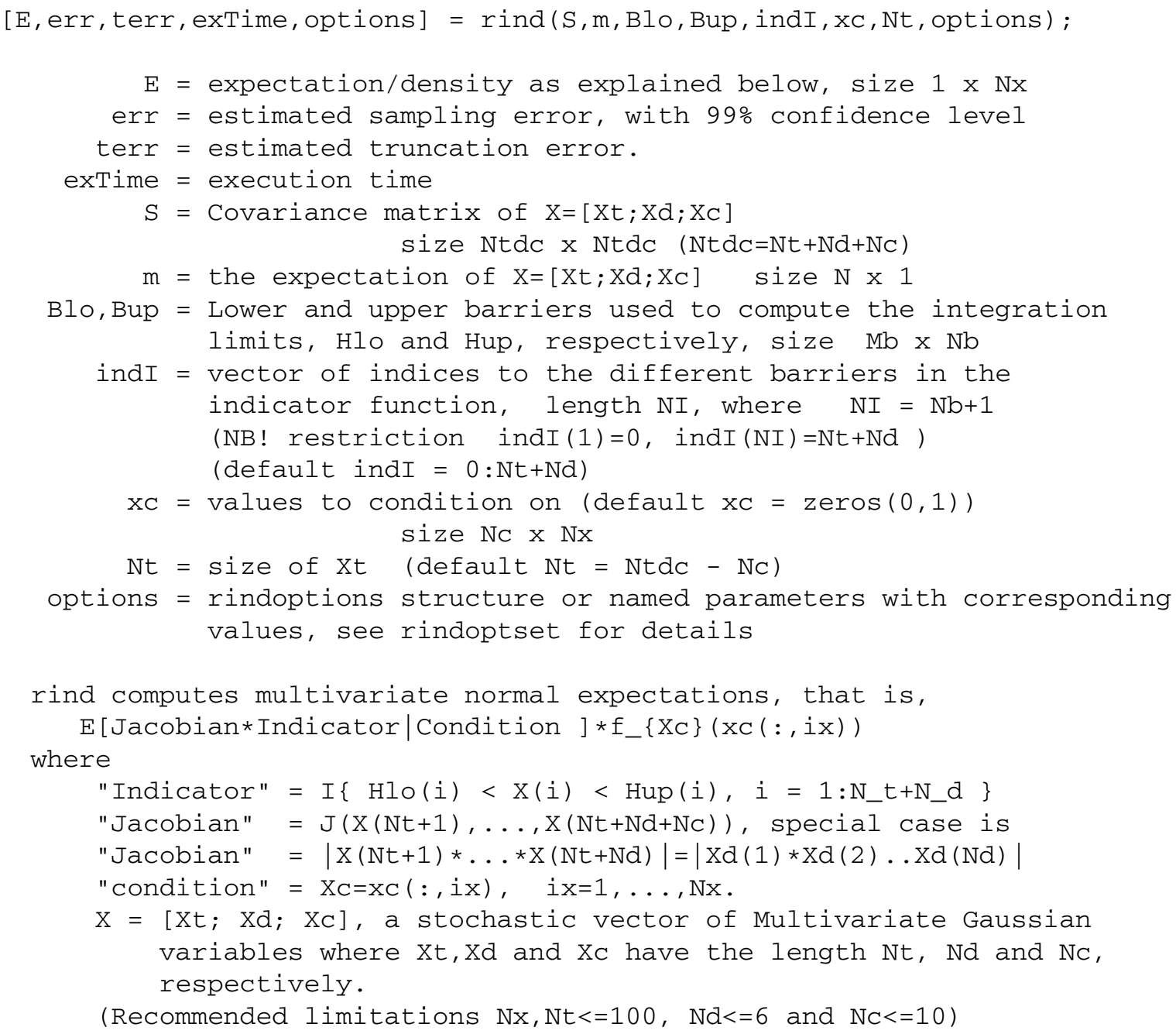




\section{ACKNOWLEDGEMENT}

I want to thank Igor Rychlik and an anonymous referee for valuable comments and criticism on the contents and presentation of an earlier version of this work.

\section{SUPPLEMENTARY MATERIAL}

Supplementary material (DOI: 10.1214/18-STS 662SUPP; .zip). The supplement contains instructions how to obtain the Wafo package, example codes to some of the figures, and additional Matlab m-files.

\section{REFERENCES}

Abrahams, J. (1982). The zero crossing problem for some nonstationary Gaussian procceses. IEEE Trans. Inform. Theory IT28 677-678.

Abrahams, J. (1986). A survey of recent progress on levelcrossing problems for random processes. In Communications and Networks (I. F. Blake and H. V. Poor, eds.) 6-25. Springer, New York.

Arellano-Valle, R. B. and Genton, M. G. (2008). On the exact distribution of the maximum of absolutely continuous dependent random variables. Statist. Probab. Lett. 78 27-35. MR2381270

Azaïs, J.-M., De Castro, Y. and Mourareau, S. (2018). Power of the spacing test for least-angle regression. Bernoulli 24 465-492. MR3706766

AzAÏs, J.-M. and GenZ, A. (2013). Computation of the distribution of the maximum of stationary Gaussian processes. Methodol. Comput. Appl. Probab. 15 969-985. MR3117635

AZAÏs, J.-M., LEÓN, J. R. and WsChEBOR, M. (2011). Rice formulae and Gaussian waves. Bernoulli 17 170-193. MR2797987

AzAÏs, J.-M. and WsCHEBOR, M. (2002). The distribution of the maximum of a Gaussian process: Rice method revisited. In In and Out of Equilibrium (Mambucaba, 2000). Progress in Probability 51 321-348. Birkhäuser, Boston, MA. MR1901961

AzAÏs, J.-M. and Wschebor, M. (2009). Level Sets and Extrema of Random Processes and Fields. Wiley, Hoboken, NJ. MR2478201

BlAKE, I. F. and LindSEY, W. C. (1973). Level-crossing problems for random processes. IEEE Trans. Inform. Theory IT-19 295-315. MR0370729

BRAININA, I. S. (2013). Applications of Random Process Excursion Analysis. Elsevier, London. MR3286421

BRODTKORB, P. A. (2006). Evaluating nearly singular multinormal expectations with application to wave distributions. Methodol. Comput. Appl. Probab. 8 65-91. MR2253077

Brodtkorb, P. A., Johannesson, P., Lindgren, G., RychLIK, I., RYDÉN, J. and SJÖ, E. (2000). WAFO—a Matlab toolbox for analysis of random waves and loads. In Proc. 10th Int. Offshore Polar Engng Conf. 3 343-350.

Cartwright, D. E. and Longuet-Higgins, M. S. (1956). The statistical distribution of the maxima of a random function. Proc. R. Soc. Lond. Ser. A 237 212-232. MR0082227

Cavanié, A., Arhan, M. and Ezraty, R. (1976). A statistical relationship between individual heights and periods of storm waves. In Proceedings, BOSS'76 354-360, Trondheim.
Cierco-Ayrolles, C., Croquette, A. and Delmas, C. (2003). Computing the distribution of the maximum of Gaussian random processes. Methodol. Comput. Appl. Probab. 5 427-438. MR2018904

CRAMÉR, H. (1940). On the theory of stationary random processes. Ann. of Math. (2) 41 215-230. MR0000920

CrAmér, H. and LeAdbetter, M. R. (1967). Stationary and Related Stochastic Processes. Wiley, New York.

Crandall, S. H. (1958). Random Vibration. Wiley and M.I.T Technology Press.

DE MARÉ, J. (1974). When are the successive zero-crossing intervals of a Gaussian process independent? Technical report, Lund Univ.

Durbin, J. (1985). The first-passage density of a continuous Gaussian process to a general boundary. J. Appl. Probab. 22 99-122. MR0776891

EFrON, B. (1997). The length heuristic for simultaneous hypothesis tests. Biometrika 84 143-157. MR1450198

Estrade, A., Iribarren, I. and Kratz, M. (2012). Chordlength distribution functions and Rice formulae. Application to random media. Extremes 15 333-352. MR2969063

GADRICH, T. and ADLER, R. J. (1993). Slepian models for nonstationary Gaussian processes. J. Appl. Probab. 30 98-111. MR1206355

GENZ, A. (1992). Numerical computation of multivariate normal probabilities. J. Comput. Graph. Statist. 1 141-149.

GENZ, A. (1993). Comparison of methods for the computation of multivariate normal probabilities. In Proc. 25th Symp. Interface Computer Science and Statistics 25 400-405.

Genz, A. (2012). Matlab software for multivariate normal probability computation. http://www.math.wsu.edu/faculty/ genz/software/software.html.

Genz, A. and Bretz, F. (2009). Computation of Multivariate Normal and $t$ Probabilities. Lecture Notes in Statistics 195. Springer, Dordrecht. MR2840595

GenZ, A. and Kwong, K.-S. (2000). Numerical evaluation of singular multivariate normal distributions. J. Stat. Comput. Simul. 68 1-21. MR1820599

Genz, A., Bretz, F., Miwa, T. and Leisch, F. (2017). Multivariate normal and $\mathrm{t}$ distribution. http://mvtnorm.R-forge. R-project.org.

GrigorIU, M. (1989). Reliability of Daniels systems subject to quasistatic and dynamic nonstationary Gaussian load processes. Probab. Eng. Mech. 4 128-134.

KAC, M. (1943). On the average number of real roots of a random algebraic equation. Bull. Amer. Math. Soc. 49 314-320. MR0007812

KAC, M. and SlePian, D. (1959). Large excursions of Gaussian processes. Ann. Math. Stat. 30 1215-1228. MR0115222

KAN, R. and Roвотті, C. (2017). On moments of folded and truncated multivariate normal distributions. J. Comput. Graph. Statist. 26 930-934. MR3765356

Khintchine, A. (1934). Korrelationstheorie der stationären stochastischen Prozesse. Math. Ann. 109 604-615. MR1512911

KRATZ, M. F. (2006). Level crossings and other level functionals of stationary Gaussian processes. Probab. Surv. 3 230-288. MR2264709

LANING, J. H. JR. and BATTIN, R. H. (1956). Random Processes in Automatic Control. McGraw-Hill, New York. MR0079362 
Lazarov, B. and Ditlevsen, O. (2005). Slepian simulation of distributions of plastic displacements of earthquake excited shear frames with a large number of stories. Probab. Eng. Mech. 20 251-262.

LeAdbetter, M. R., Lindgren, G. and Rootzén, H. (1983). Extremes and Related Properties of Random Sequences and Processes. Springer, New York. MR0691492

LINDGREN, G. (1972). Wave-length and amplitude in Gaussian noise. Adv. in Appl. Probab. 4 81-108. MR0314104

Lindgren, G. (2013). Stationary Stochastic Processes-Theory and Applications. CRC Press, Boca Raton, FL. MR3024784

LINDGREN, G. (2019). Supplement to "Gaussian integrals and Rice series in crossing distributions - to compute the distribution of maxima and other features of Gaussian processes." DOI:10.1214/18-STS662SUPP.

Lindgren, G. and BroberG, K. B. (2004). Cycle range distributions for Gaussian processes-exact and approximative results. Extremes 7 69-89. MR2201192

LINDGREN, G. and RYCHLIK, I. (1982). Wave characteristic distributions for Gaussian waves-wave-length, amplitude and steepness. Ocean Eng. 9 411-432.

LINDGREN, G. and RYCHLIK, I. (1991). Slepian models and regression approximations in crossing and extreme value theory. Int. Stat. Rev. 59 195-225.

Longuet-Higgins, M. S. (1957). The statistical analysis of a random, moving surface. Philos. Trans. R. Soc. Lond. Ser. A 249 321-387. MR0087257

Longuet-Higgins, M. S. (1962). The distribution of intervals between zeros of a stationary random function. Philos. Trans. R. Soc. Lond. Ser. A 254 557-599. MR0158431

Longuet-Higgins, M. S. (1963). Bounding approximations to the distribution of intervals between zeros of a stationary Gaussian process. In Proc. Sympos. Time Series Analysis (Brown Univ., 1962) 63-88. Wiley, New York. MR0148124

Longuet-Higgins, M. S. (1975). On the joint distribution of wave periods and amplitudes of sea waves. J. Geophys. Res. 80 2688-2694.

Longuet-Higgins, M. S. (1983). On the joint distribution of wave periods and amplitudes in a random wave field. Proc. $R$. Soc. Lond. Ser. A 389 241-258.

LYON, R. H. (1956). Response of strings to random noise fields. J. Acoust. Soc. Am. 28 391-398. MR0083342

MCFADDEN, J. A. (1958). The axis-crossing intervals of random functions. II. IRE Trans. 4 14-24. MR0098437

MERCADIER, C. (2006a). Numerical bounds for the distributions of the maxima of some one- and two-parameter Gaussian processes. Adv. in Appl. Probab. 38 149-170. MR2213969

MERCADIER, C. (2006b). MAGP (Maximum Analysis of Gaussian Processes). http://math.univ-lyon1.fr/mercadier/MAGP/index. html.

Middleton, D. (1988). S. O. Rice and the theory of random noise: Some personal recollections. IEEE Trans. Inform. Theory 34 1367-1373. MR0993430

MiLES, J. W. (1954). On the transonic drag of accelerated bodies. J. Aeronaut. Sci. 21 644-645. MR0063883

Millman, S., ed. (1984). A History of Engineering \& Science in the Bell System: Communication Sciences, 1925-1980, 1-91. AT\&T Bell Laboratories, Indianapolis, IN.

MimAKI, T. (1973). Zero-crossing intervals of Gaussian processes. J. Appl. Phys. 44 477-485.
Mimaki, T., Myoken, H. and Kawabata, T. (1985). Levelcrossing problem of a Gaussian process having Gaussian power spectrum density. Jpn. J. Appl. Phys. 24 L278-L280.

Mimaki, T., TAnabe, M. and Wolf, D. (1981). The multipeak property of the distribution densities of the level-crossing intervals of a Gaussian random process. IEEE Trans. Inform. Theory IT-27.

Munakata, T. (1998). Some unsolved problems on the level crossing of random processes. In Unsolved Problems of Noise in Physics, Biology, Electronic Technology and Information Technology (C. R. Coering, L. B. Kiss and M. F. Shlesinger, eds.) 213-222. World Scientific, Singapore.

O'HAGAN, A. (2006). Bayesian analysis of computer code outputs: A tutorial. Reliab. Eng. Syst. Saf. 91 1290-1300.

PodgóRski, K., Rychlik, I. and MAChADO, U. E. B. (2000). Exact distributions for apparent waves in irregular seas. Ocean Eng. 27 979-1016.

PodgóRski, K., Rychlik, I. and WAllin, J. (2015). Slepian noise approach for Gaussian and Laplace moving average processes. Extremes 18 665-695. MR3418772

PRÜHER, J. and SÄRKKÄ, S. (2016). On the use of gradient information in Gaussian process quadratures. In Proc. 2016 IEEE Int. Workshop on Machine Learning for Signal Processing.

RAINAL, A. J. (1988). Origin of Rice's formula. IEEE Trans. Inform. Theory 34 1383-1387. MR0993433

RAsmussen, C. E. and Williams, C. K. I. (2006). Gaussian Processes for Machine Learning. MIT Press, Cambridge, MA. MR2514435

RICE, S. O. (1944). Mathematical analysis of random noise. Bell Syst. Tech. J. 23 282-332. MR0010932

RICE, S. O. (1945). Mathematical analysis of random noise. Bell Syst. Tech. J. 24 46-156. MR0011918

Rosenbaum, S. (1961). Moments of a truncated bivariate normal distribution. J. Roy. Statist. Soc. Ser. B 23 405-408. MR0132630

RYCHLIK, I. (1987a). A note on Durbin's formula for the firstpassage density. Statist. Probab. Lett. 5 425-428. MR0903802

RYCHLIK, I. (1987b). Joint distribution of successive zero crossing distances for stationary Gaussian processes. J. Appl. Probab. 24 378-385. MR0889802

RYCHLIK, I. (1987c). Regression approximations of wavelength and amplitude distributions. Adv. in Appl. Probab. 19 396-430. MR0889943

RYCHLIK, I. (1990). New bounds for the first passage, wave-length and amplitude densities. Stochastic Process. Appl. 34 313-339. MR1047649

RYCHLIK, I. (1992a). Confidence bands for linear regressions. Comm. Statist. Simulation Comput. 21 333-352. MR1173064

RYCHLIK, I. (1992b). The two-barrier problem for continuously differentiable processes. Adv. in Appl. Probab. 24 71-94. MR1146520

SIRE, C. (2008). Crossing intervals of non-Markovian Gaussian processes. Phys. Rev. E (3) 78011121. MR2496170

SLEPIAN, D. (1962). The one-sided barrier problem for Gaussian noise. Bell Syst. Tech. J. 41 463-501. MR0133183

Slepian, D. (1963). On the zeros of Gaussian noise. In Proc. Sympos. Time Series Analysis (Brown Univ., 1962). Wiley, New York. MR0148128

Smith, J. M., Hopcraft, K. I. and Jakeman, E. (2008). Fluctuations in the zeros of differentiable Gaussian processes. Phys. Rev. E (3) 77 031112. MR2495410 
StDenis, M. and Pierson, W. J. (1953). On the Motion of Ships in Confused Seas. SNAME Trans. 61.

WAFO-GROUP (2017a). WAFO-A Matlab Toolbox for Analysis of Random Waves and Loads-Tutorial for WAFO 2017. http: //www.maths.lth.se/matstat/wafo.

WAFO-GROUP (2017b). Wafo-project. https://github.com/wafoproject.

WaX, N. (1954). Selected Papers on Noise and Stochastic Processes. Dover, New York.

WiEnER, N. (1930). Generalized harmonic analysis. Acta Math. 55 117-258. MR1555316
WiLson, L. R. M. and HopCRAFT, K. I. (2017). Periodicity in the autocorrelation function as a mechanism for regularly occurring zero crossings or extreme values of a Gaussian process. Phys. Rev. E 96062129.

WONG, E. (1966). Some results concerning the zero-crossings of Gaussian noise. SIAM J. Appl. Math. 14 1246-1254. MR0207059

Youssef, N., Munakata, T. and Takeda, M. (1996). Rice probability functions for level-crossing intervals of speckle intensity fields. Opt. Commun. 123 55-62. 